\title{
Plasticity-induced restructuring of a nanocrystalline grain boundary network
}

Jason F. Panzarino ${ }^{1}$,Zhiliang Pan $^{1}$, Timothy J. Rupert ${ }^{1,2, *}$

${ }^{1}$ Department of Mechanical and Aerospace Engineering, University of California, Irvine, CA 92697, USA

${ }^{2}$ Department of Chemical Engineering and Materials Science, University of California, Irvine, CA 92697, USA

*E-mail: trupert@uci.edu

\begin{abstract}
$\underline{\text { Abstract }}$
Thegrain boundary-mediated mechanisms that control plastic deformation of nanocrystalline metals should cause evolution of the grain boundarynetwork, since theydirectly alter misorientation relationships between crystals. Unfortunately, current experimental techniques are unable to track such evolution, due to limits on both spatial and temporal resolution. In this work, molecular dynamics simulations are used to study grain boundary restructuring in nanocrystalline $\mathrm{Al}$ during both monotonic tension and cyclic loading. This task is enabled by the creation of new analysis tools for atomistic datasets that allow for a complete characterization and tracking of microstructural descriptors of the grain boundary network. Quantitative measurements ofgrain boundary character distribution, triple junction type, grain boundaryplane normal, and other interfacial network characteristics are extracted and analyzed. The results presented here show that nanocrystalline plasticity leads to an increase in special boundary fraction and disruption of two-dimensional boundary connectivity, with the most dramatic evolution occurring in the smallest grain sizes.
\end{abstract}

Keywords: Nanocrystalline material; Cyclic micro-plasticity; Grain boundaries; Grain boundary engineering; Molecular dynamics 


\section{Introduction}

Nanocrystalline metals permanently deform through novel physicalmechanisms [1, 2] that can be attributed to an increase in the grain boundaryvolume fraction.As average grain size (d) is reduced below $\sim 10 \mathrm{~nm}$, a crossover from intragranulardislocation-based plasticity to grain boundary-mediated plasticity occurs, with the mechanisms of grain boundary sliding [3, 4], grain rotation [5-8], and grain boundary migration [9]beginning to dominate. Because the grains themselves participate, thesemechanisms are capable of altering not only grain shape but also interfacial structure during plasticity. For example,a rotating grain will change the misorientation relationship across its interfaces,potentially evolving grain boundaryconfigurations andboundary energies[10, 11].

Atomistic models, most often molecular dynamics (MD) simulations, have played a crucial role in detailing nanocrystalline deformation physics and understanding how they alter microstructure. For example, Upmanyuet al.[12] used an atomistic model of an embedded nanograin to show howsimultaneous grain rotation and grain boundary migrationreduces the overall interfacial energy of the system. Rotation rates and boundary migration velocities were highly dependent on the lattice misorientation and boundary energy, with larger misorientations and higher energies leading to relative increases in the rate of each mechanism. Similar work has also been used to uncover collective plastic rearrangement in polycrystalline systems. Hasnaouiet al.[13] used MD simulations of polycrystalline $\mathrm{Ni}(d=5 \mathrm{~nm})$ subjected to constant tensile stress and observed an alignment of interfacial shear planes through collective grain rotation caused by intergranular slip. Rupert [14]also observed collective rotation of neighboring grains in simulated $\mathrm{Ni}(d=3 \mathrm{~nm})$, reporting the development of shear localization pathways which extended across nanowire samples. A more quantitative approach was recently used by 
Panzarino et al. [15]to study relative contributions of each mechanism during mechanical cycling of polycrystalline $\operatorname{Al}(d=5 \mathrm{~nm})$. These authors noted an overall reduction in grain boundary energy, which manifested as several occurrences of rotation-induced twinning as well as coalescence of low-angle grain boundaries within the microstructure. As a whole, prior work suggests that long-range evolution of the grain boundary network is likely during plasticity for extremely fine grain sizes.

Evolution of the grain boundary network would open the door for grain boundary engineering of nanocrystalline materials,where the fraction of boundaries with"special” propertiescan be altered with thermomechanical processing[16]. One approach for identifying special boundaries is to characterize interfaces using the coincident site lattice (CSL) model [17], which assigns a value $(\Sigma)$ to each interface corresponding to the inverse of the maximum theoretical number of lattice sites which are shared between neighboring crystals, deeming those with $\Sigma \leq 29$ as special. A recent example of grain boundary engineering extended to nanocrystalline metals was presented by Bober et al. [18], who utilized transmission Kikuchi diffraction (TKD) inside of a scanning electron microscope to conclude that nanocrystalline $\mathrm{Ni}$ thin films $(d=23 \mathrm{~nm})$ subjected to thermomechanical cycling treatments will increase their $\Sigma 3$ boundary content as well as continually refine existing $\Sigma 3$ boundaries to a more perfect misorientation. This study confirmed the potential for altering grain boundaries through nanocrystalline deformation physics, but these authors focused on statistical boundary metrics rather than micro-mechanisms of evolution due to the inherent lack of temporal resolution provided by ex situcharacterization.Kobler et al.[19] began to access time-resolved measurements by using automatedcrystalorientationmappingin the transmission electron microscope (ACOM-TEM) in combination with in situ straining. These authors found evidence 
of deformation-induced grain growth, grain rotation, and twinning/detwinning in nanocrystalline Pd films with $d=37 \mathrm{~nm}$. However, because of the two-dimensional nature of data gathered by both TKD and ACOM-TEM, the orientation maps and boundary character distributions provided by such experimental techniques cannot confidently identify the grain boundaryplane orientations (CSL only requires lattice misorientation between grains). In order to fully describe a grain boundary, the boundary surface normal (two parameters) in addition to the lattice misorientation relationship between grains (threeparameters) must be determined[20].

Fortunately, since MD simulationscan provide the exact positions of atoms during the entire course of deformation, all of the required information is available to extract and observe the intricacies of grain boundary network restructuring as it is occurring, on a femtosecond timescale. Currently, such data mining is still a daunting task, even with the advent of several tools and metrics capable of quantifying nanoscale microstructural features. Several researchers have constructedanalysis methods which can identify grains[21-23], interface atom types [23], and even extract entire dislocation networks including boundary dislocations [24], but there are currently no tools that can extract and track grain boundary character from raw atomistic simulation data. In order to truly understand how grain boundary mediated plasticity can evolve grain boundary networks,new algorithms must be developed which are capable of segmenting interfacial regions and thenextractingfeatures like grain boundary surface normals, grain orientation/disorientation relationships, and triple junction structure, while simultaneouslytracking these features as they evolve throughout a simulation. Such analysis tools wouldprovide fully characterized five parameter grain boundary data with spatial and temporal resolution that is currentlyinaccessible with experimental techniques. 
In order to understand how plasticity at the nanoscale evolves grain boundary networks, we use MD simulations to subject nanocrystalline $\mathrm{Al}$ to both monotonic tension as well as mechanical cycling. Two grain sizes, $5 \mathrm{~nm}$ and $10 \mathrm{~nm}$, were examined so that differences in grain boundary network evolution associated with different plasticity mechanisms can be observed. In addition, the effect of temperature on boundary evolution during plasticity was also studied. In order to provide a truly quantitative analysis of the grain boundary network, we have developed several new techniques for characterizing and tracking grain boundary features and incorporated these tools into a recently developed analysis algorithm for atomistic datasets[21].The tool developed here is able to identify all grain boundaries, triple junctions, and vertex points while also providing interface character and network connectivity informationusing methods familiar to the experimental community. We find that special boundary fraction dramatically increases with deformation for $d=5 \mathrm{~nm}$, with elevated temperature and repetitive loading giving the largest changes. This evolution mainly manifests as an increase in the fraction of $\Sigma 3$ and $\Sigma 11$ boundaries, which we show is related to restructuring to find low-energy boundary configurations. This evolution is contrasted with boundary network evolution during thermal annealing, to highlight differences that are characteristic of mechanically-driven structural reorganization.

\section{Simulation Methods}

Sampleswithaverage grain sizes of $5 \mathrm{~nm}$ and $10 \mathrm{~nm}$ were constructed using the Voronoi method with random Euler angles assigned to each grain nucleation site [4, 25]. In addition, a minimum separation distance between grain nucleation sites was employed which allows for more equiaxed grains and a tight grain size distribution to more efficiently analyze the response 
of each specific grain size.For each sample, the same set of Euler angles was used to ensure that the starting texture and grain boundary structure would be identical despite the difference in grain size. TheLarge-scale Atomic/Molecular Massively Parallel Simulator (LAMMPS)[26]was used and all atomic interactions were described by an embedded atom method (EAM) potential for Al developed by Mishin et al. [27]. This many-body potential was developed using a combination of $a b$ initio and experimental data with the intention of simulating internal defects and plasticity in Aluminum and was found to accurately describe point defects, planar faults, grain boundaries, and experimental values of stacking fault energy.A 2 fs time step was used for time integration duringthe simulation with periodic boundary conditions applied in all directions. Any overlapping atoms which were separated by less than 2 atomic radii were removed and the final structures were then relaxed using a conjugate gradient minimization with an energy tolerance of $10^{-6} \mathrm{eV}$ and a force tolerance of $10^{-6} \mathrm{eV} / \AA$. The resulting structures were fully dense and free of stored dislocations, containing 48 grains. The $d=5 \mathrm{~nm}$ sample contained 180,982 atoms with a cubic simulation cell side length of $146.5 \AA$ and the $d=10 \mathrm{~nm}$ sample contained 1,480,503 atoms with a side length of $294.4 \AA$. The samples werethen annealedat 600 K for $100 \mathrm{ps}$ to remove excess grain boundary dislocations and free volume [28-30]. This step ensures that any observed structural evolution is not merely a byproduct of unstable high-energy interfaces caused by the construction technique used to create the samples. After equilibration,samples were cooled at a rate of $30 \mathrm{~K} / \mathrm{ps}$ until thedesired testing temperature was reached.Although these Voronoi samples are the main focus of this work, we also compare with nanocrystalline atomistic models created using two other construction techniques that give slightly different starting microstructures. Further details of this additional analysis is provided in Section 4.3. 
For mechanical testing,monotonic tension to $10 \%$ true strain and mechanical cycling were performed. Cycling was achievedthrough tension load-unload cycles. Specimens that had been loaded past the yield point to 5\% true strain were firstunloaded to 3\% strain and then pulled back to $5 \%$ strain, with each unloading-loading pairrepresenting a single cycle. This process of mechanical cycling was repeated for a total of 10 cycles.Both sets of mechanical testing simulations were run at temperatures of $300 \mathrm{~K}, 450 \mathrm{~K}$, and $600 \mathrm{~K}$, using a true strain rate of $5 \times$ $10^{8} \mathrm{~s}^{-1}$ while employing a Nose-Hoover isothermal-isobaric ensemble in order to keep zero stress in the lateral sample directions.A recent study by Zhang et al.[31]showed that, for nanocrystalline $\mathrm{Cu}$, the grain boundary mediated modes of plastic deformation are not strain rate dependent in the range of $1 \times 10^{7} \mathrm{~s}^{-1}-1 \times 10^{10} \mathrm{~s}^{-1}$. Figure 1 illustrates the deformation methodology and typical results for monotonic tension (black curve) and mechanical cycling (red curve) of the $d=5 \mathrm{~nm}$ sample at $300 \mathrm{~K}$. For monotonic tension, atomicsnapshots were output at the starting configuration and ateach strain increment of $1 \%$. For the tension unload-load cycling, simulation snapshots were recorded before deformation ( $0 \%$ strain) and each time the sample reached 5\% strain.These snapshots were subsequently analyzed using the Grain Tracking Algorithm (GTA)[21], after incorporating the improvements detailed in Section 3, and visualization of the resulting data was performed by the open source particle visualization tool OVITO[32]. The updated GTA is freely available through the corresponding author's research website or can be obtained by contacting the corresponding author.

\section{New Analysis Methods}

TheGTA was recently developedto providegrain identification, texture analysis, and grain structure evolution for atomistic simulation outputs. As outlined in a previous article that 
summarizes the initial development of the tool [21],spatial coordinates of the atoms along with a crystallinity description (e.g., centrosymmetry parameter (CSP)[33] or common neighbor analysis (CNA)[34])are all that is required to identify and track crystallites and their orientations during the simulation. CSP is a measure of the local lattice disorder surrounding an atom andwas chosen here to distinguish between crystalline and non-crystalline/defect atoms within our samples. A threshold of CSP $\geq 2.83 \AA^{2}$ was chosen to signify non-crystalline atoms in this study based on the Lindemann-Gilvarry rule[35], which establishes the maximum bond length allowable for an atom contained in a crystalline environment before melting occurs. Once atoms have been separated into crystalline and non-crystalline, the GTA calculates the lattice orientation at each crystalline position within the sample and uses this information to segment the sample into individual grains. The grain segmentation technique compares the disorientation angle (smallest symmetrically equivalent misorientation angle) between lattice orientations measured at each atomic position and compares these disorientations with an allowable cut-off angle. Any neighboring atoms which fall within this cut-off are deemed part of the same crystal. To improve grain segmentation during high temperature, all nearest neighbor vector combinations which determine a set of axis at each crystalline point are averaged to allow for more robust orientation determination. Using fcc as an example, the orientation determination procedure outlined by Panzarino et al.[21] is repeated for all 12 nearest neighbor position vectors providing 12 sets of axis which are then averaged.In addition to grain segmentation, theGTA algorithm also provides a mapping between time steps, allowing for the tracking of features such as grain size, grain rotation, and grain sliding. Figure 2(a) shows the $d=10 \mathrm{~nm}$ microstructure with atoms colored according to grain number, as well as an accompanying pole figure indicating a randomly oriented starting texture of the grains. 
Toallow forquantitative analysis and tracking of important features of the grain boundary networks, theGTA was updated in several important ways. Having calculated grain structure and orientation information, the algorithm now proceeds to distinguish between the several types of interfacial atoms within the microstructure. Boundary atoms which reside between two grain neighbors are deemed grain boundaryplane atoms, while those with three neighbors belong to triple junctions and four or more neighbors are classified as vertex point atoms. Remaining noncrystalline atoms which have only one grain neighbor (meaning they reside within the grains themselves) are deemed intragranulardefect atoms can be associated with a dislocation, stacking fault, vacancy, or interstitial. An example of this classification can be seen in Figure 2(b), where all crystalline atoms have been removed. Intragranular defect, grain boundaryplane, triple junction, and vertex atoms are colored light blue, green, yellow, and red, respectively.Examples of a stacking faultanda vacancy are labeled.Figure 2(c) displays an example of an isolated grain extracted from the computational model using the same atom classification scheme. A similar boundary atom classification was used by $\mathrm{Xu}$ and $\mathrm{Li}$ [23], but their approach obtained grain numbers and crystal orientations from the initial Voronoi construction. An outward layering methodwas then used to identify the different boundary atom types. Our method is similar in its classification scheme but also allows for the indexing of microstructures with no apriori knowledge of grain locations or orientations. Such a feature is essential for tracking boundary features during a simulation, as the material evolves.

After indexing atom types, the updatedGTA then calculates the disorientation angleand axis of rotation between all neighboringgrain pairsso that features such as $\Sigma$ type in the CSL framework can be calculated. It is important to note that the CSL framework is simply one choice for describing grain boundary character distributions and there are others such as grain 
boundary plane distribution [36]and disorientation axis distribution [37]. Since the GTA calculates all five degrees of freedom for a boundary, any of these methods is an option. We choose to use $\Sigma$ type here to describe boundary character so that our findings can be readily compared to experimental reports. The Brandon criteria[38, 39]defines the maximum allowable mis-misorientation offset, $\Delta \theta$, of an interface from a perfect $\Sigma$ misorientation relationshipand is typically defined as $\Delta \theta_{\max }=15^{\circ} / \Sigma^{1 / 2}$. Here we use the Brandon criteria, but again a simple modification the GTA script would allow for the use of other metrics. Figure 3(a) shows a nanocrystalline sample with all grain boundaryplane atoms colored according to their disorientation angle. The lighter colors indicate higher angle grain boundaries andthe boundaries highlighted in blue are special boundaries, or interfaces with $\Sigma \leq 29$ in the CSL notation.

In order to fully characterize a grain boundary and its structure,the grain boundary normal must also be determined.Since the CSL model is only related to the angle and axis which describe misorientation, each $\Sigma$ type can actually exhibit infinitely many variations depending on the dividing plane which separates the two crystals. A recent study by Homer et al.[40] illustrates the importance of boundary plane-property relationships in $\mathrm{Ni}$ and $\mathrm{Al}$ bicrystalsusing a technique developed by Patala and Schuh [41] to rewrite grain boundarynormals in terms of a symmetry-capturing fundamental zone. Their results showed that for various special boundaries,grain boundary energy and excess free volume can vary dramatically depending on the grain boundary plane orientation, with only specific boundary normals exhibiting energy minimums. This additional boundary data is essential for understanding the role of interface planes ingrain boundary network evolution and several other emerging studies also highlight the importance of a complete grain boundary character analysis which includes such information [42-44]. 
To obtain the grain boundarynormal, the updated GTAsegments the grain boundaryplane atoms into planar sections based on whether or not each atom and its neighbors constitute a relatively flat section, which allows for the identification of faceted boundaries. First, local surface normals are calculated at each grain boundary atom using a singular value decomposition to find a best-fit plane foreach atom and a surrounding cluster of its nearest neighbors.Singular value decomposition determines a plane which minimizes the square sum of the orthogonal distances between the points and the best-fit plane. For nanocrystalline microstructures, a cluster of50grain boundary neighbor atoms appropriately capturesaccurate interface normals at each atom, with an example shown in Figure 3(b).This process is repeated for all of the atoms in the boundary and a normal is assigned to every atomic position. When building up agrain boundary section,a grain boundary normal variation angle, $\theta_{n}$, must be selected which provides a maximum allowable normal variation between neighboring atoms. Figure 3(b)illustrates this concept by showing three neighboring atoms with their normal vectors assigned and how their orientations can vary by $\theta_{n}$. A convergence study was performed to find an adequate grain boundary cutoff angle. The grain boundary network of the $10 \mathrm{~nm}$ average grain size sample $(T=450 \mathrm{~K})$ was analyzed at $10 \%$ strain while varying $\theta_{n}$ from $4-12^{\circ}$ and recording the amount of grain boundary atoms which were unable to be allocated to grain boundary sections. The results of the convergence study can be seen in Figure 3(c).A value of $8^{\circ}$ allows for reasonable accuracy and the ability tosegment flat sections of grain boundary, while also ensuring that there are only a small numberof non-allocated interface atoms.For stricter cutoff angles, many atoms withingrain boundary planeswould be unallocated. Alternatively, larger cutoff angles would not adequately segment curved interfaces into planar sections.Proper selection of this normal cutoff angle is important for nanocrystalline systems with very smallgrain sizes, where planar grain boundary 
sections tend to be very small and grain boundaries can be highly curved.With boundary plane normal identified, allfivegrain boundary parameters in an atomistic sample can be fully characterized.

With boundaries completely described, higher level topological features such as triple junctions can also be quantified. Depending upon how many of the connecting interfaces exhibit special character, triple junctions can be assigned a designation number ortriple junction type. Since connectivity of high energy interfaces also has implications regarding percolation of fracture-susceptible boundaries $[45,46]$, several past studies have focused efforts on characterizingtriple junction typedistribution andclusterconnectivity using two-dimensional computational models or orientation imaging microscopy scans[47-50], with a threedimensionalnumerical studyutilizing atetrakaidecahedramodel for the grain shapes [51]. In order to be consistent withthis previous work, triple junction types are assigned here as follows: junctions with no attached special boundaries are deemed type 0 , junctions with one special boundary are called type 1, and so on. Figure 3(d) shows the triple junction network in the $d=$ $10 \mathrm{~nm}$ sample where the atoms are color coded by type number. Analyzing the density as well as connectivity of these special interface types can help illuminate the role of triple junctiontopology during microstructural evolution.

\section{Microstructural Evolution During Plastic Deformation}

\subsection{Monotonic Loading}

First, the effect of simple monotonic plasticity on the grain boundary network is studied.During deformation, evolution of the grain boundarycharacter distribution was analyzed 
and special boundary fractions are reported.The fraction of boundary atoms which are detected as having $\Sigma \leq 29$ is plotted as a fraction of the total number of grain boundaryplane atoms. Since this metric is an atomic fraction, our calculation will be similar to length fraction, but with an added grain boundary thickness component which we expect to have minimal effect.Grain boundary special fractionsare presented in Figure 4(a) and 4(b), as a function of applied strain, for the $=5 \mathrm{~nm}$ and $d=10 \mathrm{~nm}$ samples, respectively. As the $5 \mathrm{~nm}$ grain size samples are deformed, the fraction of special boundaries shows some fluctuations, with the general trend that the special fraction increases during the majority of the plastic deformation range.These increases in special fractionoccurred through both an increase in the length of existing special boundaries as well as the development of new special boundaries that were not present before deformation. Occasional jumps in the data are a result of large boundaries which evolve into or out of special character as additional grain rotation occurs, so it is most instructive to look at general trends. Most obvious is the fact that, tensionat higher temperatures led to faster increases to the special boundary fraction. Further inspection showed thatboundaries with low potential energy were more likely to cease rotation and hold a consistent disorientation relationship after their formation. For example, $\Sigma 3$ boundaries became sessileif $\{111\}$ boundary planes were formed. However, other $\Sigma 3$ boundaries with randomly oriented boundary planes were not guaranteed to keep their character through the rest of the deformation process and occasionally moved away from a special configuration. Similar behavior was observed for other special boundaries as well. This importance of grain boundary plane on boundary stability highlights the power of being able to extract such information from atomistic datasets, a capability enabled by the updated GTA. 
For the $10 \mathrm{~nm}$ samples, the grain boundary special fractionremained relatively constant with some fluctuations around the starting value.This difference can be attributed to the larger grain size,where dislocation-based mechanisms start to dominate.Schiotzand coworkers[52, 53] noted an inverse Hall-Petch relationship in the flow stress of simulated $\mathrm{Cu}$ when average grain size fell below 10nm and further inspection showed that plastic strain was highly concentrated at the grain boundaries in the finest grained samples.Kadauet al.[54] alsoshowed that a transition inplasticity mechanisms occurs nearthis grain size for Voronoi-created Al samples tested by MD.In our analysis, grain rotation is found to be significantly restricted for the larger grain size and an increase in the density of residual stacking faults left in the microstructure was observed for the $10 \mathrm{~nm}$ sample, which are both consistent with a shift toward dislocation-based plasticity mechanisms. In addition, no significant reduction in the potential energy of special boundaries was measured during deformation of the larger grain size sample.

The accompanying triple junction network was also tracked, withFigure 4(c) and (d)showing the evolution of type 0 triple junctions as a function of applied strain. The measurement of triple junction fraction was carried out in a fashion similar to special boundary fraction, with the total number of atoms residing in each triple junction type divided by the total number of triple junction atoms present. The decrease in type 0 triple junction fraction for the $=$ $5 \mathrm{~nm}$ sample is a direct consequence of the increase in specialboundary fraction with applied plastic strain. With more and more special boundaries, it becomes less likely that a triple junction will not have a special boundary attached to it. Because of the limited change in special fraction for the $d=10 \mathrm{~nm}$ sample, there was no clear trend in type 0 evolution. The remaining triple junction types were also analyzed, but no obvious trends were found and no additional 
insight was obtained through the metric that could not otherwise be portrayed in the special fraction data.

Connectivity and topology of the boundary network was also analyzed to understand if any long-range evolution isoccurring. Since it is known that connectivity of the grain boundary network relates to intergranular failure such as fracture and corrosion[46, 55-59], the size of connected "random boundary clusters" of non-special boundary atomswas measured.Figure 5 compares a two-dimensionalslice of the $d=5 \mathrm{~nm}$ sample at strains of $1 \%$ and $10 \%$ at $600 \mathrm{~K}$. Special boundaries, triple junction types greater than zero, and vertex points which adjoin these special features were removed from the slices, since specialinterfaces tend to be less susceptible to intergranular failure.The remaining grain boundary atoms are segmented into clusters and colored using the cluster analysis tool developed by Stukowski[32] which groups clusters of particles using a user-specified cutoff radius, with a cluster radius of $6 \AA$ used for Figure 5.A comparison ofFigure 5(a) and (b) shows that there is significant break-up in the random boundary cluster size with increased plastic strain. This means that, for example, a crack moving from left to right through the microstructure would not be able to find a path which only propagatesalong random, non-special boundaries. The connectivity of random boundary clusters in two-dimensions is commonly reported in the experimental literature for grain boundary engineering (see, e.g.,[16, 60]).However, extension to three-dimensional analysis, available in our simulation method but not generally available from experiments, shows no large changes in cluster number or average size.Even though the network is broken up locally along a twodimensional slice, the additional dimensionality allows for more percolation pathways and any breakups can be potentially bypassed through another route present in the grain boundary network. In fact, Figure 5(c) is the same sample at $10 \%$ strain with blue atoms illustrating the 
single three dimensional cluster that exists despite the fact that the special boundaries (green), triple junctions of type greater than zero (yellow), and vertex points (red) were removed from the cluster analysis.Again, this observation highlights the utility of atomistic modeling for analysis of boundary network features with additional detail.

\subsection{Cyclic Loading}

According to Fig. 4(a), the majority of special fraction increase occurred within the intermediate 3-8\% strain rangeand higher temperatures magnify this effect. In an attempt to drive further reconstruction of the grain boundary network, mechanical cycling in the range of 35\% true strain was simulated.Figures 6(a) and (b) plot the special boundary atomicfractions, with the X-axis beginning at the starting configuration of $0 \%$ strain (labelled "Start") and then showing data from the end of each cycle (Cycle 0 is the initial loading to $5 \%$ strain). It is clear from Figure 6(a)that cycling at higher temperature gives a faster increase in specialboundary fraction for the $d=5 \mathrm{~nm}$ sample. In fact, cycling at $300 \mathrm{~K}$ does not clearly alter the special fraction in an obvious manner. Like monotonic loading, theobserved evolutionis due to both an increase in the length of existing special boundaries as well as the emergence of new special boundaries. Figure 6(b) shows the special boundary fraction evolution for thed $=10 \mathrm{~nm}$ grain size as a function of cycle number. There is a noticeable decrease in the special boundary fractionduring the $600 \mathrm{~K}$ cycling, as well as a small decrease for cycling at $300 \mathrm{~K}$ and $450 \mathrm{~K}$. Generally, there is significantly less evolution for the larger grain size.For the $5 \mathrm{~nm}$ grain size, it is instructive to compare the special boundary fraction achieved through mechanical cycling to that obtained with monotonic tension. By the 10 th cycle, the $d=5 \mathrm{~nm}$ sample tested at $600 \mathrm{~K}$ contains a 0.175 fraction of special boundaries (or an increase of $\sim 75 \%$ from the starting value) 
whereas simple tension only resulted in a fraction of 0.12 or less for all strainsat or below $5 \%$. Even considering all possible strains up to $10 \%$, monotonic tension only results in a 0.14 special boundary fraction.While monotonictension and mechanical cycling bothincrease the special boundary fraction in these samples, cyclic loadingallows for extra boundary rearrangement to occur.

The main difference ingrain boundarynetwork evolution between these two grain sizes can be attributed to increased activation of grain rotation in the smaller grain size sample,as shown in Figure 6(c) where average grain rotation is plotted as a function of cycle number for the $600 \mathrm{~K}$ simulations. Here, grain rotation is measured as the angular disorientation of a grain from its starting configuration (Start), before any deformation was imposed. As a whole, the $d=$ $5 \mathrm{~nm}$ sample experienced roughly three times as much grain rotation as the $10 \mathrm{~nm}$ grain size sample. It is also important to note that these values are averaged over all grains in the sample. For example, there were several grains in the $d=5 \mathrm{~nm}$ sample which rotated more than 5 degrees, but none in thed $=10 \mathrm{~nm}$ sample. The error bars in Figure 6(c) show the standard deviation of the grain rotation angles. This grain rotation was often accompanied bysimultaneous grain boundary migration of known high-energy interfaces.For example, noncoherent sections of $\Sigma 3$ boundaries migrated to allow for lengthening of coherent $\Sigma 3$ sections.Thistype of evolution was found to occur for other types of special boundaries as well. Figure 7 displays a more detailed picture of the resulting evolution for allspecial boundaries in the $d=5 \mathrm{~nm}$ and $d=10 \mathrm{~nm}$ samples that were mechanically cycled at $600 \mathrm{~K} . \Sigma 13, \Sigma 17, \Sigma 19$, $\Sigma 21, \Sigma 25, \Sigma 27$, and $\Sigma 29$ boundaries each have "a" and "b" variations that were tabulated but these are reported here in a combined fashion using their respective $\Sigma$ value. Variations in CSL types are a result of specific $\Sigma$ boundaries which have multiple disorientation angle/axis pairs 
which result in the same reciprocal coincident site density[61]. Figure 7(a) indicates a distinct increasein $\Sigma$ 3and $\Sigma 11$ boundaries, while other $\Sigma$ types show no clear trend or even slight decreases (see the $\Sigma 15$ population). In contrast, Figure 7(b) illustrates noticeably lower special fraction evolution within the $d=10 \mathrm{~nm}$ microstructure during cycling and lack of a clear trend.

It is widely reported that special boundaries can exhibit low energy,but this is really only rigorously true for certain interface plane normal values[11, 62]. Additionally, several computational works[12, 15, 63] and experimentalstudies[64, 65] provide evidence that grain rotation is a mechanism by which interfacial energy can be reduced.Therefore, one explanation for the increase in $\Sigma 3$ and $\Sigma 11$ boundaries is thatrotation-mediated boundary rearrangement allows for the emergence of these special boundary types once favorable grain boundary planes are achieved.Early experimental works studied rotation-induced formation of specialboundaries through the use of single crystalparticles sintered to flat plates[65, 66]. Since the particles were sitting freely on top of the substrate, they were unconstrained by any neighboring crystals and thus free to rotate during sintering. Herrmann et al. [65]discovered the strong emergence of $\Sigma 3$ as well as $\Sigma 11$ boundariesduring the sintering of both $\mathrm{Cu}$ and $\mathrm{Ag}$ particles in this type of experiment.Because our present study shows high levels of grain rotation ford $=5 \mathrm{~nm}$, thissuggeststhat nano-grains are able to rotate to low-energy cusps in the boundary energy landscape in a similar fashion.

Analysisofseveral specific special boundary types is presented in Figure 8(a), where the average atomic potential energy is plotted versus cycle number. The remaining special boundary types that are not shown follow roughly the same trend as the $\Sigma 5$ boundaries. The average potential energies of crystalline atoms as well as randomboundary atoms are also presented as grey and black data points, respectively. Even before loading of any kind, the pre-existing $\Sigma 3$ 
and $\Sigma 11$ boundary atoms have lower energy than other grain boundary atoms, foreshadowing the restructuring to come that will increase their fraction within the microstructure. The $\Sigma 3$ boundary atoms approach a lower energy state with added cycles, especially after the initial pull and first cycle. Additional analysis of the $\Sigma 3$ boundariesshows that the average mismisorientation, or angular deviation from the perfect CSL disorientation,decreases with cycling, meaning that they are becoming closer to a perfect $\Sigma 3$ misorientation. This mis-misorientation evolution of $\Sigma 3$ boundaries for the $=5 \mathrm{~nm}$ samplesis shown in Figure 8(b). The majority of the $\Sigma$ boundary planes evolve to coherent $\{111\}$ planes by the $10^{\text {th }}$ cycle, while those that did not were the facets that enabled $\{111\}$ plane formation elsewhere. Visual evidence of this is shown in Figure 8(c),which displays all $\Sigma 3$ boundaries at Cycle 10 for the $d=5 \mathrm{~nm}$, $600 \mathrm{~K}$ sample. In contrast, the average energy of all $\Sigma 11$ boundaries was relatively lowin the original configuration and only fluctuated slightly with cycling. The $\Sigma 5, \Sigma 9, \Sigma 27$ boundaries did not undergo significant evolution, with their energies roughly matching the average atomic energy of the remaining random boundaries. Data for the $\Sigma 9$ and $\Sigma 27$ boundariesdoes not appear across all cycles because they were not always present in the sample. Finally there is even some slight reduction in the average energy of crystalline atoms which can probably be attributed to structural relaxation of the system during early loading.

Knowing that $\Sigma 3$ and $\Sigma 11$ boundaries were evolving to reduce interfacial energy, site specific occurrences of the formation of these boundary types were thenstudied to obtain more insight into the underlying restructuring mechanisms. As an example, Figure 9(a)-(c) follows the structural rearrangement and formation of four special boundaries which all border a common grain. For clarity, the crystalline atoms as well as triple junction and vertex point atoms have been removed. By Cycle 4, three of the interfaces have become special, having evolved from 
previously random boundary types. The details of each misorientation relationship are listed below each snapshot and color coded according to the respective boundary. After first listingthe $\Sigma$ value, the disorientation angle between grains and the mis-misorientation angle are provided. Next, the rotation axis is listed, followed by the grain boundary normal vector that is written in terms of the crystal orientations of the two neighboring grains. For those boundaries which are highly curved, the normal vectors are not listed until an adequately planar interface or faceted

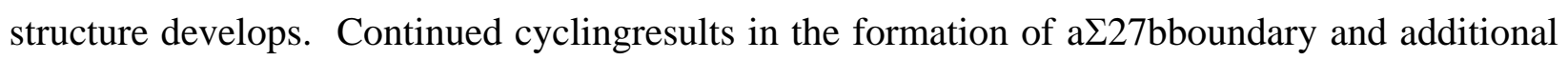
structural rearrangement of the interfaces. First, the $\Sigma 3$ boundary reduces its mis-misorientation from $5.5^{\circ}$ to $1.89^{\circ}$ by the last cycle. Cycling also allowed for an increase in the surface area of the $\Sigma 3$ interface. The $\Sigma 25$ b, which did not have an ordered boundary plane, shrinks during cycling. The $\Sigma 27 \mathrm{~b}$ forms at the end of cycle 7,but there is little change in mis-misorientation with continued cycling. The most interesting evolution is observed for the $\Sigma 11$ boundary.This boundary begins to facet during cycling,to reduce the boundary energy at the cost of increased surface area.Facets are denoted in Figure 9(b) and (c) using dashed lines. Further inspection of the grain boundary energy and facet structure evolution is shown in Figure 9(d)-(f), which is a slightly rotated view of the $\Sigma 11$ boundary colored by potential energy. Three facets at the end of Cycle 8 are denoted by black arrows and have low relative potential energy, especially at the innermost atomic layer. The facet steps continue to restructure during the next two cycles, resulting in additional length of the low energy segments. Figure $9(\mathrm{~g})$ shows the detection of the facet planes as computed by the GTA with character information listed below the image.These low energy planes are $\Sigma 11\{113\}$ grain boundary sections, which are known to be a minimum energy cusp for symmetric tilt boundaries[40]. 


\subsection{Effect of Starting Configuration}

As some recent studies have questioned whether a Voronoi grain structure is a realistic representation of real polycrystalline microstructure [67-71], it is nature to ask whether the results reported above are general findings or related specifically to the evolution of a Voronoi grain structure. To rule out any artifacts due to any specific sample generation technique, our originalVoronoisamples were compared to two additional samples generated from separatethreedimensionalisotropic grain growth models developed by Lazar et al.[72] and Syhaand Weygand[73], with a focus on cycling of the $d=5 \mathrm{~nm}$ structures at $600 \mathrm{~K}$ since this was the condition that showed the most evolution. These models allow for different, potentially more realistic, distributions of grain size as well as grain boundary topological features and boundary curvatures that may not be present within an as-assembled Voronoi construction.The FrontTracking implementation of Lazar et al.[72]is known to reproduceinteresting geometrical features such as two-sided faces and three-faced bodies, which occur as the transitioning microstructure coarsens to a steady state.The Vertex Dynamics model of Syha and Weygand[73] includes an additional force term which incorporates the effects of inclination and misorientation dependent grain boundary energy. Both models satisfy the MacPherson-Srolovitzrelation for grain growth rates in three dimensions[74]. Lazar et al. [72] and Syhaand Weygand[73]developed coarsened microstructures by starting with grains that were initially generated using theVoronoi construction and then grown using their Front-Tracking and Vertex Dynamics algorithms, respectively. In the present study, we have taken the grain and boundary locations from these two studies and filled them with atoms corresponding to randomly oriented crystals using the open source code NanoSCULPT developed by Prakash et al. [75]. The overall sample size was scaledtomicrostructures that are comparable to our as-assembledd $=5 \mathrm{~nm}$ grain 
size, beforethen subjecting both of these samples to the $600 \mathrm{~K}$ anneal for $100 \mathrm{ps}$. These two samples were then cycled at $600 \mathrm{~K}$ in order to observe and compare the resulting grain boundary network evolution.For the remainder of this section our three different starting configurations will be referred to asVoronoi, Vertex Dynamics, and Front-Tracking to facilitate comparison between the different sample generation methods.

Visual comparisons of all three starting configurations can be seen in Figure 10(a)-(c) which are sliced along a (111) sample plane and color coded according to grain identification by the GTA. The Vertex Dynamics and Front-Tracking samples contain larger numbers of grains,which allows for improved evolution statistics as well as some insight into whether ourprevious results are affected by the selected sample size. The Vertex Dynamics sample contained 573,690 atoms with a cubic simulation cell measuring $146.5 \AA$ in length and the FrontTracking sample contained 632,469 atoms with a cubic simulation cell measuring $222.0 \AA$. The grain size distribution for all three samples is presented in Figure 10(d), where there is a clear contrast between the sharp distribution in theVoronoi sample as compared to the broader distributions found within the grain growth models. The exact average grain sizes for all three models after the $600 \mathrm{~K}$ annealwerecomputed to be $5.4 \mathrm{~nm}, 6.7 \mathrm{~nm}$, and $6.4 \mathrm{~nm}$ for the Voronoi, Vertex Dynamics, and Front-Tracking samples, respectively, based on an equivalent spherical diameter calculated from the atomic volume of eachGTA identified grain.

Special boundary and $\Sigma 3$ fraction evolution is shown in Figure 11(a) for all three sample generation methods as a function of cycle number. Even with a varying initial distribution of special boundary content there is a consistent increase in special boundary fraction during cycling for all $\Sigma \leq 29$. The $\Sigma 3$ content evolves slightly fasterin the Voronoi samples, which could in part be attributed to the slightly smaller average grain size and an increased contribution 
from grain rotation.The slight reduction in $\Sigma 3$ evolution may be connected to the fact that the wider grain size distribution of the Vertex Dynamics and Front Trackingcontain a few grains inthe $10 \mathrm{~nm}$ range, where we previously reported less $\Sigma$ fraction evolution in Figure 7(b).In the end though, significant evolution of both special boundary and $\Sigma 3$ fractions are observed with cycling, demonstrating that the observations made in this paper are applicable to a general nanocrystalline grain structure. The complete evolution of each special boundarytype is presented in Figure 11(b) and (c) forthe Vertex Dynamics and Front-Tracking, respectively. An interesting trend across the two new samples is the increase in $\Sigma 3$ and $\Sigma 11$ boundary fractions, similar to the Voronoi observations. The three models produce differing initial $\Sigma$ fractions, meaning that some details of the grain boundary character evolution are different. For example, the Front-Tracking sample has the largest fraction of $\Sigma 5$ boundaries. Since these are relatively high energy boundaries and the system evolves to a lower energy state during cycling, the $\Sigma 5$ fraction rapidly decreases during cycling. As another example, the Vertex Dynamics sample has an increasing $\Sigma 13$ fraction, while maintaining the relatively large initial fraction of $\Sigma 29$ and $\Sigma 21$ boundaries. As a whole though, the comparison of the three different samples allows general conclusions to be drawn. Mechanical cycling at elevated temperature allows the grain structure to rearrange, with an increase in low energy $\Sigma 3$ and $\Sigma 11$ fractions in all cases.

\subsection{Comparison with evolution during annealing}

The results shown in the prior two sections clearly show that plasticity is capable of restructuring nanocrystalline grain boundary networks so that lower energy configurations can be found. Thermal annealing is another common way to drive microstructural evolution toward a lower energy state, and can serve as a comparison point for the mechanically-induced 
restructuring already described. To facilitate such a comparison, thed $=5$ and $10 \mathrm{~nm}$ samples were also subjected to thermal treatments at $800 \mathrm{~K}$.The $600 \mathrm{~K}$ equilibration samples were heated in a linear fashion to $800 \mathrm{~K}$ over the course of $100 \mathrm{ps}$, then held at this temperature for 1 ns with atomistic snapshots analyzed in 100 ps time intervals. The grain boundaryspecial fractionsduring this annealing are presented in Figure 12(a). There was adramatic increase in special boundary fraction for the $5 \mathrm{~nm}$ grain size, butno increasefor the $d=10 \mathrm{~nm}$ sample. An indepth analysis of boundary evolution in the $5 \mathrm{~nm}$ sample is shown in Figure 12(b). Most noticeable is the rapid increase in $\Sigma 3$ boundary fraction during annealing.After reaching $800 \mathrm{~K}$ ( 0 ps),partial dislocation emission along successive $\{111\}$ planes allows for pre-existing stacking faults to form into small annealing twins. These twinned regions remain fixed as the remaining grain boundaries within the samplemigrate rapidly in directions parallel to the $\{111\}$ planes, to lengthen the coherent twin interfaces. The majority of the $\Sigma 3$ content was observed to develop in this manner with minor evolution occurring to the preexisting non-coherent $\Sigma 3$ boundaries, the majority of which disappeared from the microstructure soon after heating.Bringa et al. [76] discovered five-fold twinning in simulated $\mathrm{Cu}(d=5 \mathrm{~nm})$ annealed at $800 \mathrm{~K}$, explaining that high local stresses within the grain boundaries combined with elevated temperature can allow for emission of twinning partials during annealing.Although lengthening of $\Sigma 3$ boundaries dominatesnetwork evolution during annealing, some small increase to the $\Sigma 5, \Sigma 7$, and $\Sigma 11$ population also occurs. Unlike the mechanical loading case where $\Sigma 11$ boundaries are the second most frequent special boundary, $\Sigma 5$ content increases most quickly during annealing. However, after $\sim 500$ ps the average grain size becomes very coarse and several grains approach the length of our simulation cell. 
In order to show the typical physics of network restructuring during annealing,a representative example of $\Sigma 3$ boundary lengtheningis shown in Figure 13(a)-(c). As the annealing simulation begins, the highly curved, random boundaries start tomigrate toward their centers of curvature (black arrows). This migration allows a coherent (111)interface to increase its length dramatically and obvious grain growth has occurred by 200 ps. Further inspection shows that this is not the only special boundary which formed along this grain pairduring the annealing process. Figure 13(d)presents a side view showing the formation of a second twin boundary and a $\Sigma 7$ boundary. A facet step present in Twin 1 is also observed. Additional annealing allowedfor the continued migration of adjacent random boundary segments, which lengthens the $\Sigma 3$ boundaries and $\Sigma 7$ boundary until they meet and lock in positionat 500 ps, as shown in Figure 13(e). The $\Sigma 7$ boundary obtains a distinct (111) orientation normal by this time, which is a minimum energy configuration for the $\Sigma 7$ boundary type[40]. Network rearrangement during annealing therefore leads to different special boundaries ( $\Sigma 5$ and $\Sigma 7$ are common) and different mechanisms of evolution (curvature-driven grain growth).

\section{Conclusions}

In this work, a quantitative analysis of the grain boundary network and its evolution during monotonic and cyclic plastic deformation was presented. Since experimental techniques lack the temporal and spatial resolution for such measurements, molecular dynamics were used to simulate deformation while new analytical tools are created to quantify important grain boundary features. By investigating two grain sizes that span the Hall-Petch breakdown, we find that finer nanocrystalline grain sizes experience more evolution of their grain boundary network 
during plasticity. In addition, themagnitude ofnetwork restructuring ishighly temperature dependent. Several important conclusions can be drawn from this work:

- Analysis tools were developed that allow forquantification of the grain boundary network in atomistic models. The five degrees of freedom associated with any grain boundary section can be measured, as well as features associated with triple junctions and vertex points. With this information, grain boundary character distributions and network topology/connectivity can be characterized with nanometer and femtosecond resolution.

- Both monotonic and cyclic plasticity drive an increase in the special boundary fraction for $d=5 \mathrm{~nm}$. The majority of the increase was associated with higher $\Sigma 3$ and $\Sigma 11$ boundary fractions, through both the lengthening of existing boundaries and the creation of new boundary sections. For the $\Sigma 3$ boundaries specifically, mis-misorientation decreases during cycling and low-energy (111) boundary planes are often found. Similar faceting to create low-energy $\Sigma 11$ interfaces is also observed. The preference for these low-energy grain boundary types suggests that the grain structure is rearranging to reduce system energy and find a more stable configuration.

- Negligible restructuring was found for the $d=10 \mathrm{~nm}$ sample. This lack of evolution can be linked to the reduced activation of collective plastic mechanisms such as grain rotation and the increase in dislocation activity.

- Even when varying the method used to create the starting microstructure,which also alters the initial distribution of special boundary content, a consistent increase in $\Sigma \leq 29$ fraction is found for small grain sizes during mechanical cycling at elevated temperature. The formation of low energy $\Sigma 3$ and $\Sigma 11$ boundaries is common to all samples. 
- Annealing also leads to a reduction in system energy, but there are important differences in the special boundary types which form and in the mechanisms for formation. $\Sigma 3$ boundaries lengthen by curvature-driven grain growth, where fast migration of random boundaries parallel to the (111) twin planes drastically increases $\Sigma 3$ content.

This study shows that the collective deformation physics associated with nanocrystalline metals can lead to evolution and restructuring of the grain boundary network. Grain boundary networks are thereforevery dynamic at the finest nanoscale grain sizes and grain boundary engineering through new mechanisms is an intriguing possibility for these materials.

\section{Acknowledgements}

We gratefully acknowledge support from the National Science Foundation through a CAREER Award No. DMR-1255305.The authors also thank Dr. Daniel Weygand forsupplying the Vertex Dynamics microstructure and Dr. Emanuel Lazar for supplying the Front-Tracking microstructure, which were then used to create atomistic samples in this work. 


\section{REFERENCES}

[1] K.S. Kumar, H. Van Swygenhoven, S. Suresh. Mechanical behavior of nanocrystalline metals and alloys, Acta Mater. 51 (2003) 5743-5774.

[2] M.A. Meyers, A. Mishra, D.J. Benson. Mechanical properties of nanocrystalline materials, Prog. Mater. Sci. 51 (2006) 427-556.

[3] H. Van Swygenhoven, P.A. Derlet. Grain-boundary sliding in nanocrystalline fcc metals, Phys. Rev. B 64 (2001) 224105.

[4] J. Schiotz, F.D. Di Tolla, K.W. Jacobsen. Softening of nanocrystalline metals at very small grain sizes, Nature 391 (1998) 561-563.

[5] J. Schiotz, T. Vegge, F.D. Di Tolla, K.W. Jacobsen. Atomic-scale simulations of the mechanical deformation of nanocrystalline metals, Phys. Rev. B 60 (1999) 11971-11983.

[6] Z.W. Shan, E.A. Stach, J.M.K. Wiezorek, J.A. Knapp, D.M. Follstaedt, S.X. Mao. Grain boundarymediated plasticity in nanocrystalline nickel, Science 305 (2004) 654-657.

[7] M. Ke, S.A. Hackney, W.W. Milligan, E.C. Aifantis. Observation and measurement of grain rotation and plastic strain in nanostructured metal thin-films, Nanostruct. Mater. 5 (1995) 689-697.

[8] L. Wang, J. Teng, P. Liu, A. Hirata, E. Ma, Z. Zhang, M.W. Chen, X. Han. Grain rotation mediated by grain boundary dislocations in nanocrystalline platinum, Nat. Commun. 5 (2014) 5402.

[9] D. Farkas, A. Froseth, H. Van Swygenhoven. Grain boundary migration during room temperature deformation of nanocrystalline Ni, Scr. Mater. 55 (2006) 695-698.

[10] A. Haslam, S. Phillpot, D. Wolf, D. Moldovan, H. Gleiter. Mechanisms of grain growth in nanocrystalline fcc metals by molecular-dynamics simulation, Mater. Sci. Eng., A 318 (2001) 293-312.

[11] D.L. Olmsted, S.M. Foiles, E.A. Holm. Survey of computed grain boundary properties in facecentered cubic metals: I. Grain boundary energy, Acta Mater. 57 (2009) 3694-3703.

[12] M. Upmanyu, D.J. Srolovitz, A.E. Lobkovsky, J.A. Warren, W.C. Carter. Simultaneous grain boundary migration and grain rotation, Acta Mater. 54 (2006) 1707-1719.

[13] A. Hasnaoui, H. Van Swygenhoven, P.M. Derlet. Cooperative processes during plastic deformation in nanocrystalline fcc metals: A molecular dynamics simulation, Phys. Rev. B 66 (2002) 184112.

[14] T.J. Rupert. Strain localization in a nanocrystalline metal: Atomic mechanisms and the effect of testing conditions, J. Appl. Phys. 114 (2013) 033527.

[15] J.F. Panzarino, J.J. Ramos, T.J. Rupert. Quantitative tracking of grain structure evolution in a nanocrystalline metal during cyclic loading, Modell. Simul. Mater. Sci. Eng. 23 (2015) 025005.

[16] C.A. Schuh, M. Kumar, W.E. King. Analysis of grain boundary networks and their evolution during grain boundary engineering, Acta Mater. 51 (2003) 687-700.

[17] H. Grimmer, W. Bollmann, D.H. Warrington. Coincidence-Site Lattices and Complete PatternShift Lattices in Cubic-Crystals, Acta Crystallogr., Sect. A A 30 (1974) 197-207.

[18] D.B. Bober, M. Kumar, T.J. Rupert. Nanocrystalline grain boundary engineering: Increasing Sigma 3 boundary fraction in pure Ni with thermomechanical treatments, Acta Mater. 86 (2015) 43-54.

[19] A. Kobler, A. Kashiwar, H. Hahn, C. Kubel. Combination of in situ straining and ACOM TEM: A novel method for analysis of plastic deformation of nanocrystalline metals, Ultramicroscopy 128 (2013) 68-81.

[20] P. Lejcek. Grain boundary segregation in metals, Springer Science \& Business Media, 2010.

[21] J.F. Panzarino, T.J. Rupert. Tracking Microstructure of Crystalline Materials: A Post-Processing Algorithm for Atomistic Simulations, JOM 66 (2014) 417-428.

[22] G.J. Tucker, S.M. Foiles. Molecular dynamics simulations of rate-dependent grain growth during the surface indentation of nanocrystalline nickel, Mater. Sci. Eng., A 571 (2013) 207-214. 
[23] T. Xu, M. Li. Geometric methods for microstructure rendition and atomic characterization of poly- and nano-crystalline materials, Philos. Mag. 90 (2010) 2191-2222.

[24] A. Stukowski, V.V. Bulatov, A. Arsenlis. Automated identification and indexing of dislocations in crystal interfaces, Modell. Simul. Mater. Sci. Eng. 20 (2012) 085007.

[25] G. Voronoi. New applications of continuous parameters to the theory of quadratic forms. - First memoir - Some properties of perfect positive quadratic forms, J. Reine. Angew. Math. 133 (1908) 97178.

[26] S. Plimpton. Fast Parallel Algorithms for Short-Range Molecular-Dynamics, J. Comput. Phys. 117 (1995) 1-19.

[27] Y. Mishin, D. Farkas, M. Mehl, D. Papaconstantopoulos. Interatomic potentials for monoatomic metals from experimental data and ab initio calculations, Phys. Rev. B 59 (1999) 3393.

[28] G.J. Tucker, D.L. McDowell. Non-equilibrium grain boundary structure and inelastic deformation using atomistic simulations, Int. J. Plast. 27 (2011) 841-857.

[29] T.J. Rupert, C.A. Schuh. Mechanically driven grain boundary relaxation: a mechanism for cyclic hardening in nanocrystalline Ni, Philos. Mag. Lett. 92 (2012) 20-28.

[30] A. Hasnaoui, H. Van Swygenhoven, P.M. Derlet. On non-equilibrium grain boundaries and their effect on thermal and mechanical behaviour: a molecular dynamics computer simulation, Acta Mater. 50 (2002) 3927-3939.

[31] T. Zhang, K. Zhou, Z. Chen. Strain rate effect on plastic deformation of nanocrystalline copper investigated by molecular dynamics, Mater. Sci. Eng., A 648 (2015) 23-30.

[32] A. Stukowski. Visualization and analysis of atomistic simulation data with OVITO-the Open Visualization Tool, Modell. Simul. Mater. Sci. Eng. 18 (2010) 015012.

[33] C.L. Kelchner, S.J. Plimpton, J.C. Hamilton. Dislocation nucleation and defect structure during surface indentation, Phys. Rev. B 58 (1998) 11085-11088.

[34] D. Faken, H. Jónsson. Systematic analysis of local atomic structure combined with 3D computer graphics, Comput. Mater. Sci. 2 (1994) 279-286.

[35] J.J. Gilvarry. The Lindemann and Gruneisen Laws, Phys. Rev. 102 (1956) 308-316.

[36] X. Liu, D. Choi, H. Beladi, N.T. Nuhfer, G.S. Rohrer, K. Barmak. The five-parameter grain boundary character distribution of nanocrystalline tungsten, Scr. Mater. 69 (2013) 413-416.

[37] M.N. Kelly, K. Glowinski, N.T. Nuhfer, G.S. Rohrer. The five parameter grain boundary character distribution of $\alpha$-Ti determined from three-dimensional orientation data, Acta Mater. 111 (2016) 22-30.

[38] D.G. Brandon. Structure of High-Angle Grain Boundaries, Acta Metall. 14 (1966) 1479-1484.

[39] A.H. King, S. Shekhar. What does it mean to be special? The significance and application of the Brandon criterion, J. Mater. Sci. 41 (2006) 7675-7682.

[40] E. Homer, S. Patala, J. Priedeman. Grain Boundary Plane Orientation Fundamental Zones and Structure-Property Relationships, Sci. Rep. 5 (2015) 15476.

[41] S. Patala, C.A. Schuh. Symmetries in the representation of grain boundary-plane distributions, Philos. Mag. 93 (2013) 524-573.

[42] A. King, G. Johnson, D. Engelberg, W. Ludwig, J. Marrow. Observations of intergranular stress corrosion cracking in a grain-mapped polycrystal, Science 321 (2008) 382-385.

[43] K. Matsunaga, H. Nishimura, S. Hanyu, H. Muto, T. Yamamoto, Y. Ikuhara. HRTEM study on grain boundary atomic structures related to the sliding behavior in alumina bicrystals, Appl. Surf. Sci. 241 (2005) 75-79.

[44] W.Z. Han, M.J. Demkowicz, E.G. Fu, Y.Q. Wang, A. Misra. Effect of grain boundary character on sink efficiency, Acta Mater. 60 (2012) 6341-6351.

[45] V. Gertsman, M. Janecek, K. Tangri. Grain boundary ensembles in polycrystals, Acta Mater. 44 (1996) 2869-2882. 
[46] T. Watanabe. The Impact of Grain-Boundary-Character-Distribution on Fracture in Polycrystals, Mater. Sci. Eng., A 176 (1994) 39-49.

[47] C.A. Schuh, R.W. Minich, M. Kumar. Connectivity and percolation in simulated grain-boundary networks, Philos. Mag. 83 (2003) 711-726.

[48] C.A. Schuh, M. Kumar, W.E. King. Universal features of grain boundary networks in FCC materials, J. Mater. Sci. 40 (2005) 847-852.

[49] J. Basinger, E. Homer, D. Fullwood, B. Adams. Two-dimensional grain boundary percolation in alloy 304 stainless steel, Scr. Mater. 53 (2005) 959-963.

[50] R.W. Minich, C.A. Schuh, M. Kumar. Role of topological constraints on the statistical properties of grain boundary networks, Phys. Rev. B 66 (2002) 052101.

[51] M. Frary, C.A. Schuh. Connectivity and percolation behaviour of grain boundary networks in three dimensions, Philos. Mag. 85 (2005) 1123-1143.

[52] J. Schiotz. Atomic-scale modeling of plastic deformation of nanocrystalline copper, Scr. Mater. 51 (2004) 837-841.

[53] J. Schiotz, K.W. Jacobsen. A maximum in the strength of nanocrystalline copper, Science 301 (2003) 1357-1359.

[54] K. Kadau, T.C. Germann, P.S. Lomdahl, B.L. Holian, D. Kadau, P. Entel, M. Kreth, F. Westerhoff, D.E. Wolf. Molecular-dynamics study of mechanical deformation in nano-crystalline aluminum, Metall. Mater. Trans. A 35a (2004) 2719-2723.

[55] D.B. Wells, J. Stewart, A.W. Herbert, P.M. Scott, D.E. Williams. The Use of Percolation Theory to Predict the Probability of Failure of Sensitized, Austenitic Stainless-Steels by Intergranular StressCorrosion Cracking, Corrosion 45 (1989) 649-660.

[56] L.C. Lim, T. Watanabe. Fracture-toughness and brittle-ductile transition controlled by grainboundary character distribution (GBCD) in polycrystals, Acta Metall. Mater. 38 (1990) 2507-2516.

[57] G. Palumbo, P.J. King, K.T. Aust, U. Erb, P.C. Lichtenberger. Grain-Boundary Design and Control for Intergranular Stress-Corrosion Resistance, Scr. Metall. Mater. 25 (1991) 1775-1780.

[58] V.Y. Gertsman, K. Tangri. Modelling of intergranular damage propagation, Acta Mater. 45 (1997) 4107-4116.

[59] Y. Ikuhara, T. Watanabe, T. Yamamoto, T. Saito, H. Yoshida, T. Sakuma. Grain boundary structure and sliding of alumina bicrystals, Mater. Res. Soc. Symp. P. 601 (2000) 125-132.

[60] S. Tsurekawa, S. Nakamichi, T. Watanabe. Correlation of grain boundary connectivity with grain boundary character distribution in austenitic stainless steel, Acta Mater. 54 (2006) 3617-3626.

[61] D. Brandon. The structure of high-angle grain boundaries, Acta Metall. 14 (1966) 1479-1484.

[62] K. Merkle, D. Wolf. Low-energy configurations of symmetric and asymmetric tilt grain boundariest, Philos. Mag. A 65 (1992) 513-530.

[63] J.W. Cahn, J.E. Taylor. A unified approach to motion of grain boundaries, relative tangential translation along grain boundaries, and grain rotation, Acta Mater. 52 (2004) 4887-4898.

[64] U. Erb, H. Gleiter. Effect of Temperature on the Energy and Structure of Grain-Boundaries, Scr. Metall. 13 (1979) 61-64.

[65] G. Herrmann, H. Gleiter, G. Baro. Investigation of Low-Energy Grain-Boundaries in Metals by a Sintering Technique, Acta Metall. 24 (1976) 353-359.

[66] S.-W. Chan, R. Balluffi. Study of energy vs misorientation for grain boundaries in gold by crystallite rotation method-I.[001] twist boundaries, Acta Metall. 33 (1985) 1113-1119.

[67] T. Xu, M. Li. Topological and statistical properties of a constrained Voronoi tessellation, Philos. Mag. 89 (2009) 349-374.

[68] D. Gross, M. Li. Constructing microstructures of poly-and nanocrystalline materials for numerical modeling and simulation, Appl. Phys. Lett. 80 (2002) 746-748. 
[69] M. Li, T. Xu. Topological and atomic scale characterization of grain boundary networks in polycrystalline and nanocrystalline materials, Prog. Mater. Sci. 56 (2011) 864-899.

[70] A. Leonardi, P. Scardi, M. Leoni. Realistic nano-polycrystalline microstructures: beyond the classical Voronoi tessellation, Philos. Mag. 92 (2012) 986-1005.

[71] A. Leonardi, M. Leoni, P. Scardi. Atomistic modelling of polycrystalline microstructures: An evolutional approach to overcome topological restrictions, Comput. Mater. Sci. 67 (2013) 238-242.

[72] E.A. Lazar, J.K. Mason, R.D. MacPherson, D.J. Srolovitz. A more accurate three-dimensional grain growth algorithm, Acta Mater. 59 (2011) 6837-6847.

[73] M. Syha, D. Weygand. A generalized vertex dynamics model for grain growth in three dimensions, Modell. Simul. Mater. Sci. Eng. 18 (2010) 015010.

[74] R.D. MacPherson, D.J. Srolovitz. The von Neumann relation generalized to coarsening of threedimensional microstructures, Nature 446 (2007) 1053-1055.

[75] A. Prakash, M. Hummel, S. Schmauder, E. Bitzek. Nanosculpt: A methodology for generating complex realistic configurations for atomistic simulations, MethodsX 3 (2016) 219-230.

[76] E.M. Bringa, D. Farkas, A. Caro, Y.M. Wang, J. McNaney, R. Smith. Fivefold twin formation during annealing of nanocrystalline Cu, Scr. Mater. 59 (2008) 1267-1270.

\section{FIGURES}

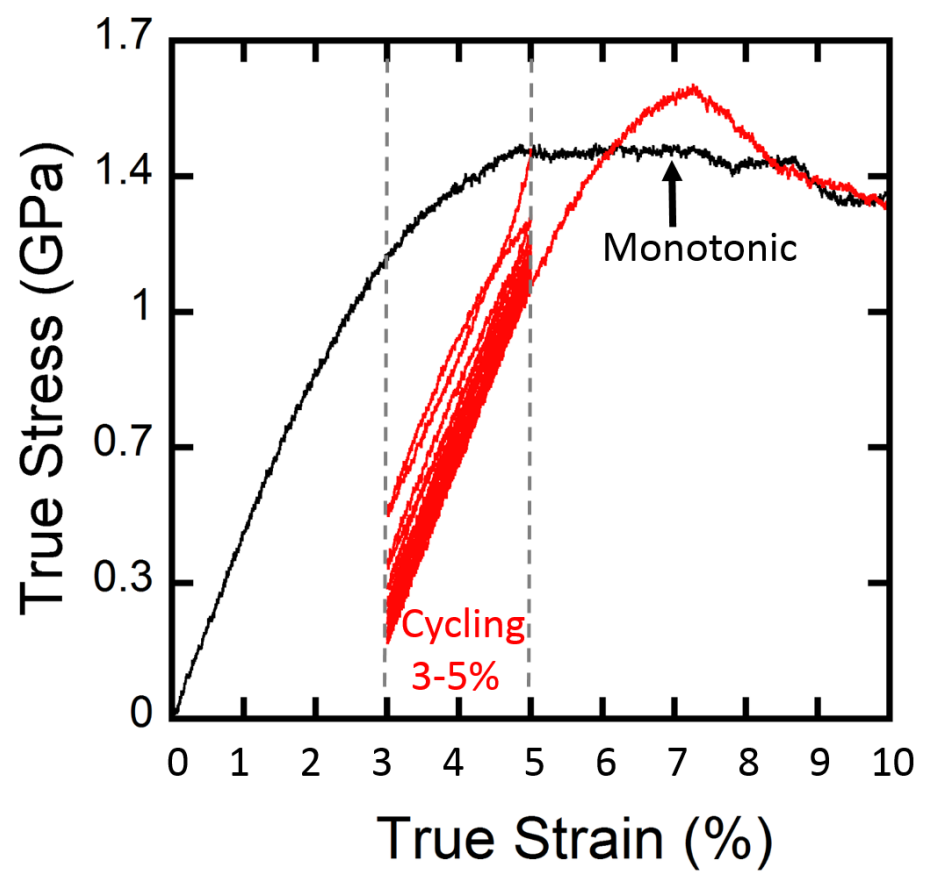

Figure 1. Mechanical response of $d=5 \mathrm{~nm}$ sample loaded monotonically (black line) and cyclically (red line) at $300 \mathrm{~K}$. Dotted grey lines illustrate the mechanical cycling procedure. Identical loading procedures were performed at $450 \mathrm{~K}$ and $600 \mathrm{~K}$. 


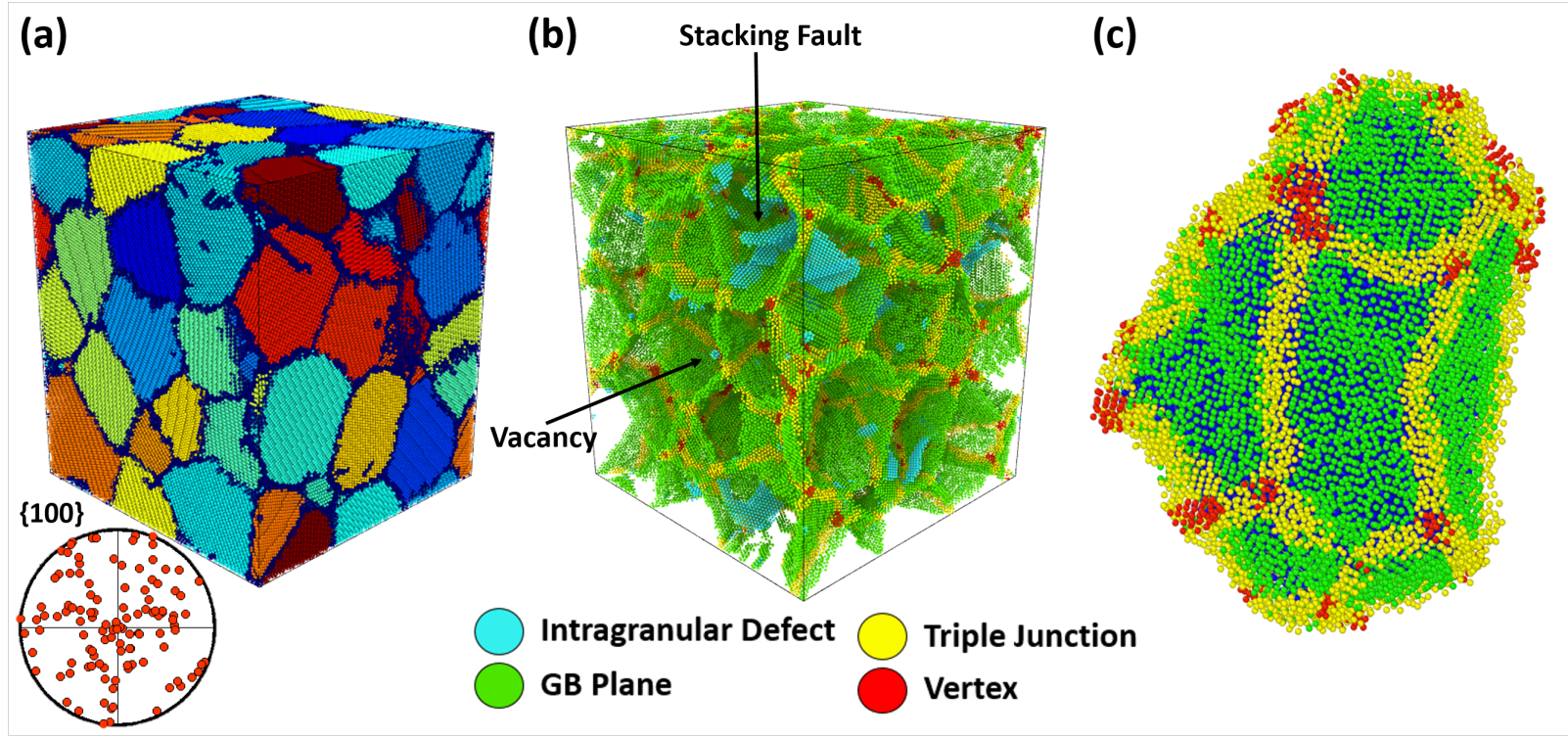

Figure 2. (a) The $d=10 \mathrm{~nm}$ sample at $10 \%$ true strain and $600 \mathrm{~K}$ with all grain identified. The $\{100\}$ pole figure indicates random texture throughout the sample, even after tensile deformation. (b) The grain boundary and intragranular defect network of the same sample with atoms colored according to defect type. Blue atoms are intragranular defects (stacking fault, void, or interstitial) while green, yellow, and red atoms are grain boundary plane, triple junction, and vertex points, respectively. (c) A single grain surrounded by the various grain boundary network atom types. 
(a)
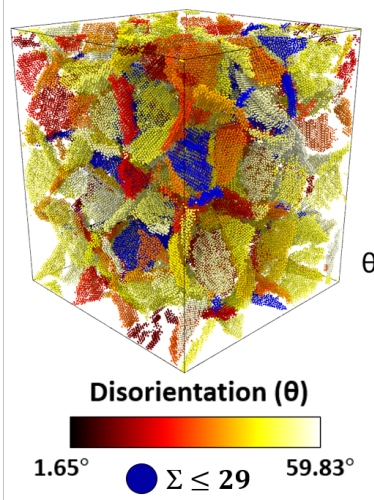

(b)

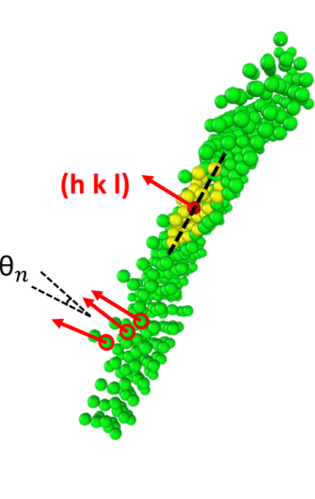

(c)

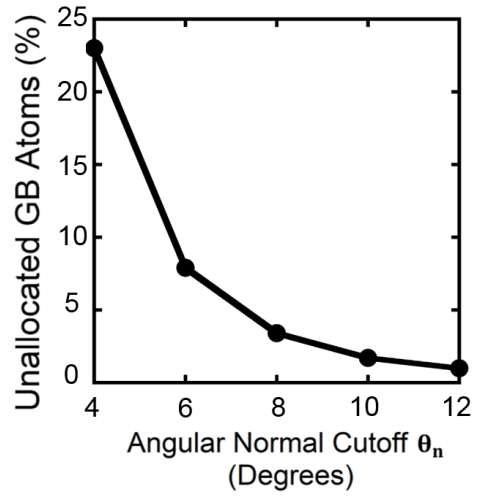

(d)

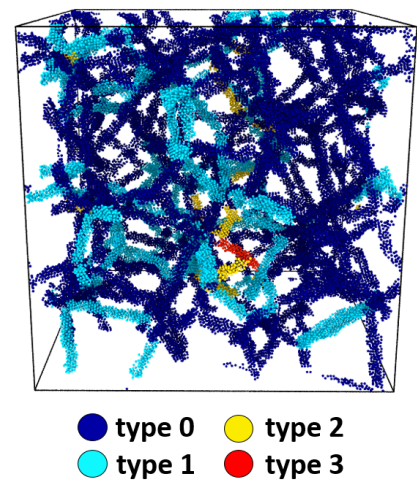

Figure 3. (a) Grain boundary plane atoms colored according to disorientation angle. Special boundaries are highlighted in blue. (b) Example of a cluster of grain boundary plane atoms where a specific atom (red) and 50 of its neighbors (yellow) were used to calculate a local plane normal. This process is repeated until all grain boundary interface atoms are assigned a normal vector. Neighboring normal vectors must deviate by less thana specified angular cutoff value in order to be considered part of the same boundary plane. (c) The effect of varying the angular cutoff on the number of grain boundary interface atoms which are allocated to planar sections. (d) The triple junction network colored according to junction type. 


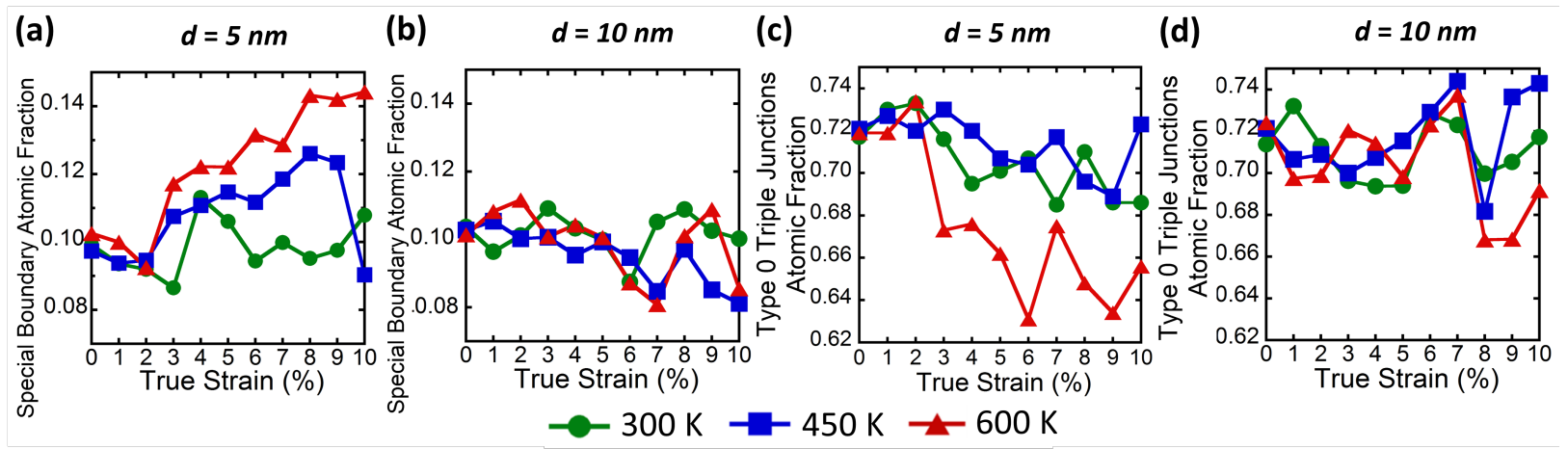

Figure 4. The evolution of special boundary fraction for (a) $d=5 \mathrm{~nm}$ and (b) $d=10 \mathrm{~nm}$ monotonic tension tests at all testing temperatures. (c) Type 0 triple junction analysis for $d=5 \mathbf{n m}$ sample, showing evolution which was inversely proportional to the special boundary fraction evolution. (d) Type 0 triple junction fraction for the $d=10 \mathrm{~nm}$ grain size exhibits fluctuations, but no discernable upward or downward trend. 

(a) $\varepsilon=1 \%$

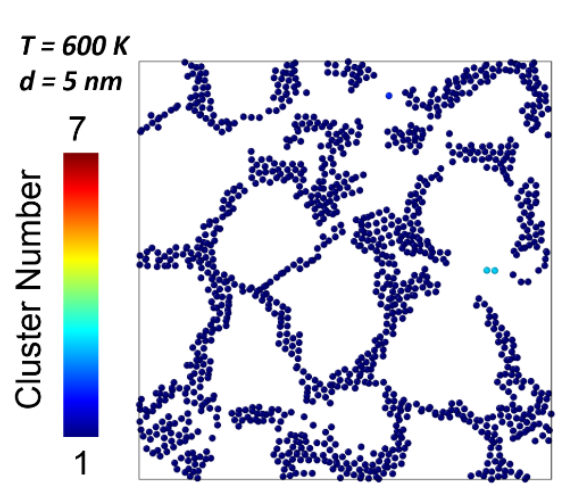

(b) $\varepsilon=10 \%$

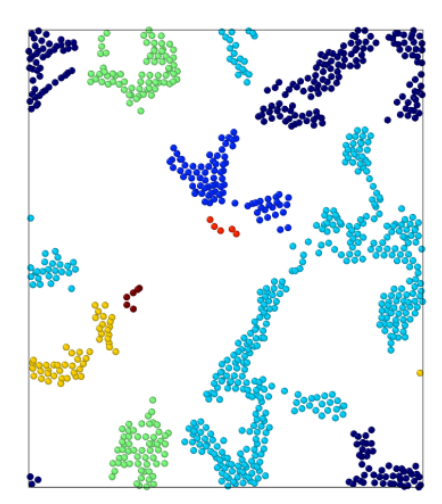

(c) $\varepsilon=10 \%$

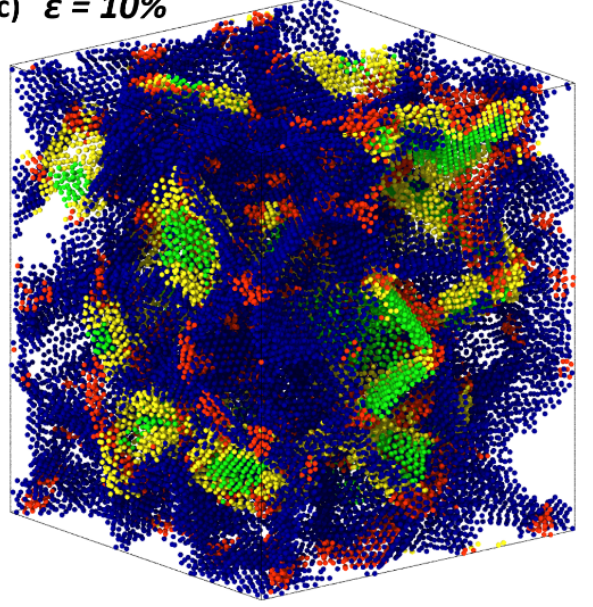

Figure 5. Two-dimension cluster analysis of the $d=5 \mathrm{~nm}$ grain boundary network. For both (a) and (b) only those atoms which are not special nor are part of a special junction type are analyzed, with the colors associated with different boundary clusters. After being pulled to $10 \%$ strain, a clear breakup in the random network is observed.(c) The same sample with special boundaries (green), triple junctions (yellow) and vertex points (red) which were removed from the cluster analysis. The remaining atoms(blue) make up a single cluster which maintains connectivity throughout the sample. 


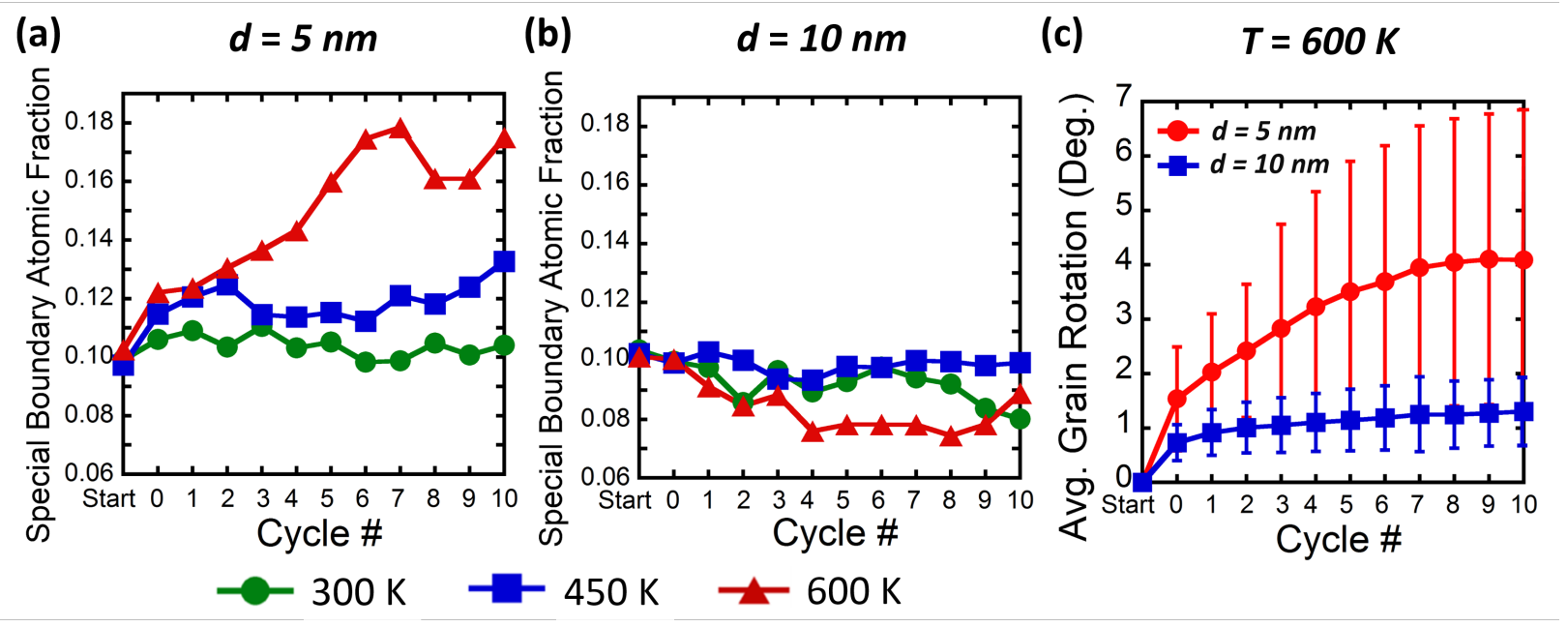

Figure 6. Special boundary fraction of mechanically cycled samples for (a) $d=5 \mathbf{n m}$ and (b) $d=10$ $\mathrm{nm}$ for all testing temperatures. (c) Average grain rotation, measured as the disorientation from a grain's starting configuration, for $5 \mathrm{~nm}$ and $10 \mathrm{~nm}$ average grain sizes cyclically loaded at $600 \mathrm{~K}$. 


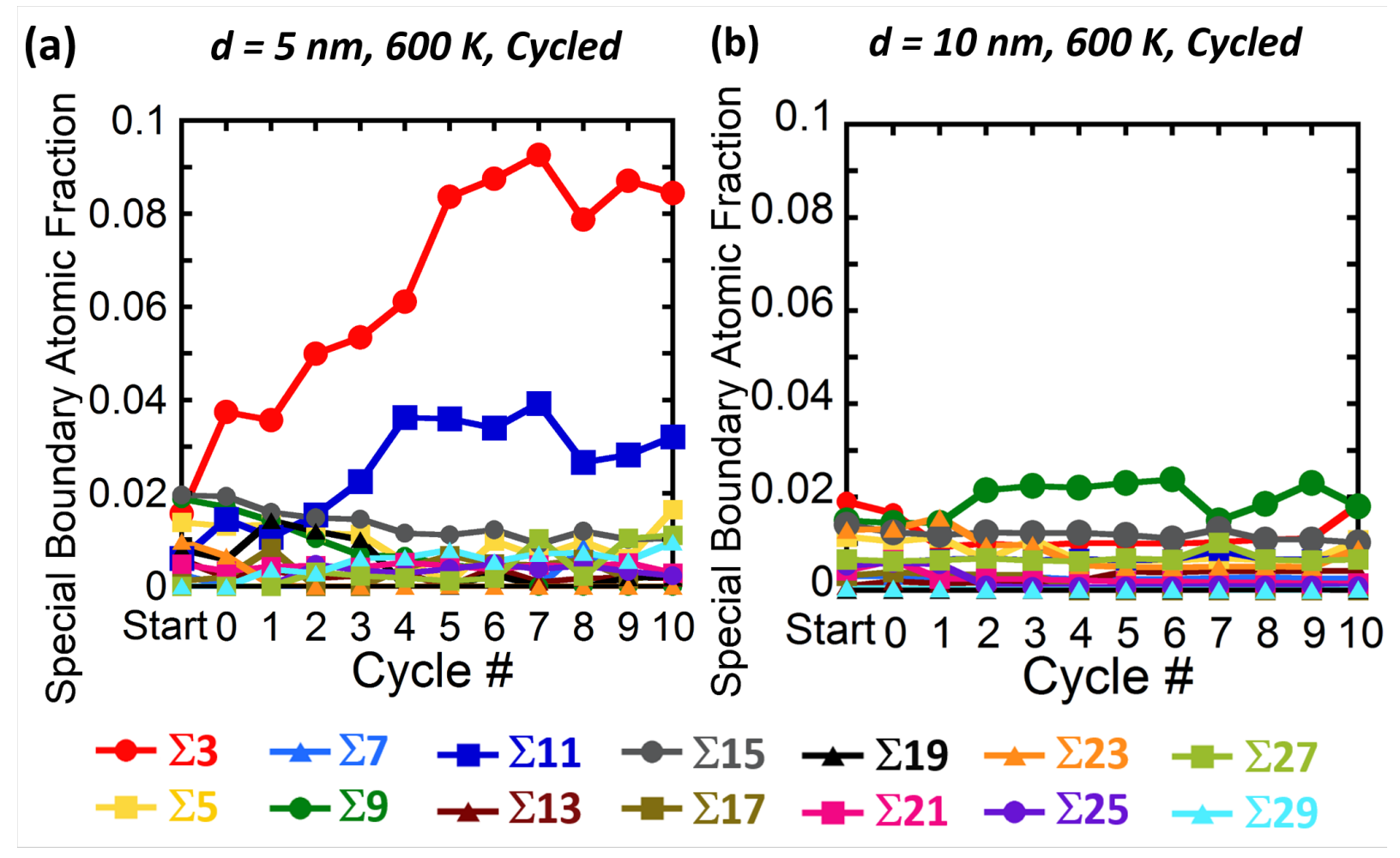

Figure 7. Special boundary fraction for the (a) $d=5 \mathrm{~nm}$ at $600 \mathrm{~K}$ sample shows an increase in $\Sigma 3$ and $\Sigma 5$ boundary content. (b) The $d=10 \mathrm{~nm}$ samples experiencevery little evolution of the special boundary fraction during cycling. 
(a)

$$
d=5 \mathrm{~nm}, 600 \mathrm{~K}
$$

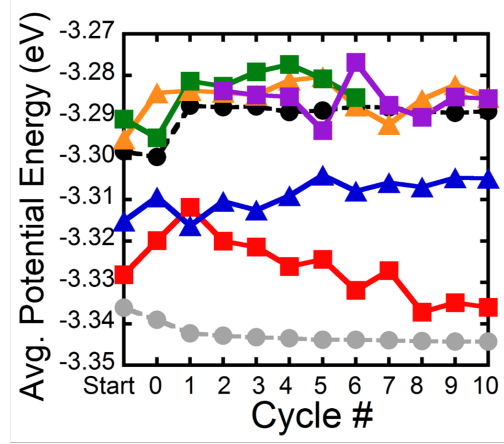

(b) $\quad d=5 \mathrm{~nm}$

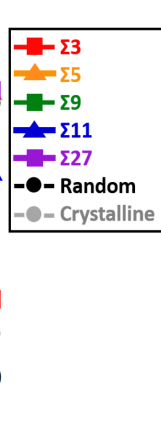

(c) $d=5 \mathrm{~nm}, 600 \mathrm{~K}$, Cycle 10

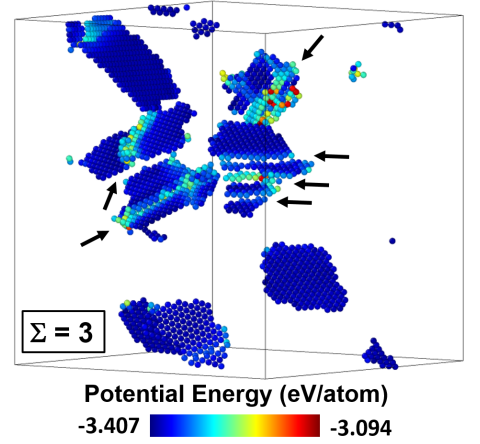

Figure 8. (a) Average potential energies of select special boundary atoms, random boundary atoms, and crystalline atoms during cycling. (b) Mis-misorientation of $\Sigma 3$ boundaries as a function of cycle number. (c) $\Sigma 3$ boundaries at Cycle 10, with black arrows indicating facet steps which allowed coherent segments to form. 


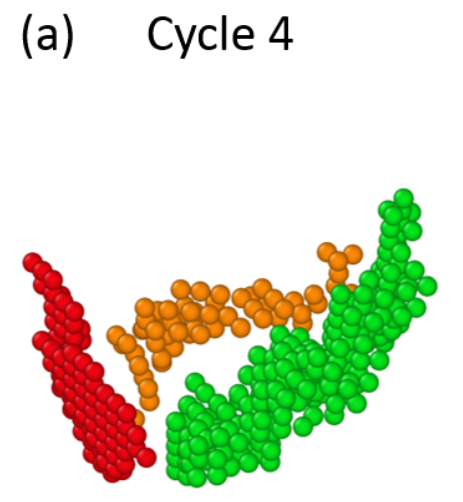

$\Sigma 355.7^{\circ} / 5.5^{\circ}[111](111) /(111)$

$\Sigma 1147.7^{\circ} / 4.3^{\circ}[110]$

$\Sigma 25 \mathrm{~b} 48.6^{\circ} / 2.86^{\circ}[331]$ (b)

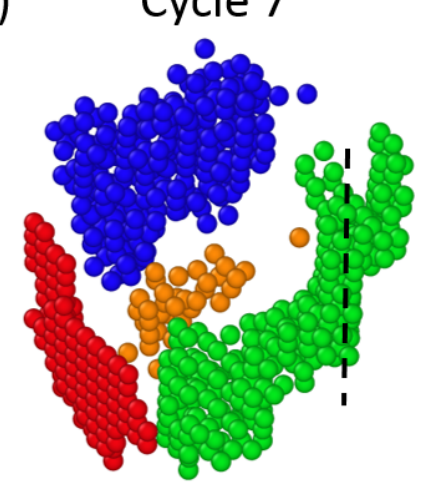

$\Sigma 356.5^{\circ} / 4.1^{\circ}[111](111) /(111)$

$\Sigma 1148.8^{\circ} / 3.9^{\circ}[110]$

$\Sigma 25 \mathrm{~b} 49.3^{\circ} / 2.4^{\circ}[331]$

$\Sigma 27 \mathrm{~b} 37.6^{\circ} / 2.6^{\circ}[111][111](111) /(12 \overline{1})$ (c) Cycle 10

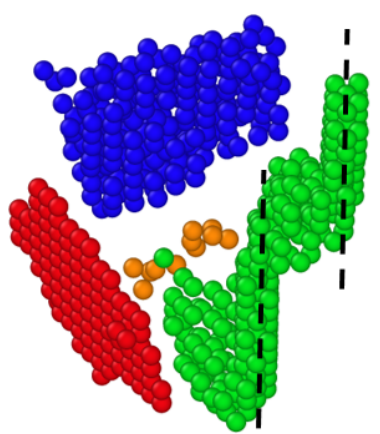

$\Sigma 358.45^{\circ} / 1.89^{\circ}[111](111) /(111)$

$\Sigma 1150.4^{\circ} / 3.7^{\circ}[110]$

$\Sigma 25 \mathrm{~b} 49.9^{\circ} / 2.9^{\circ}[331]$

$\Sigma 27 \mathrm{~b} 36.5^{\circ} / 2.8^{\circ}[111](111) /(12 \overline{1})$

(d) Cycle 8

(e) Cycle 9

(f) Cycle 10

(g) Cycle 10
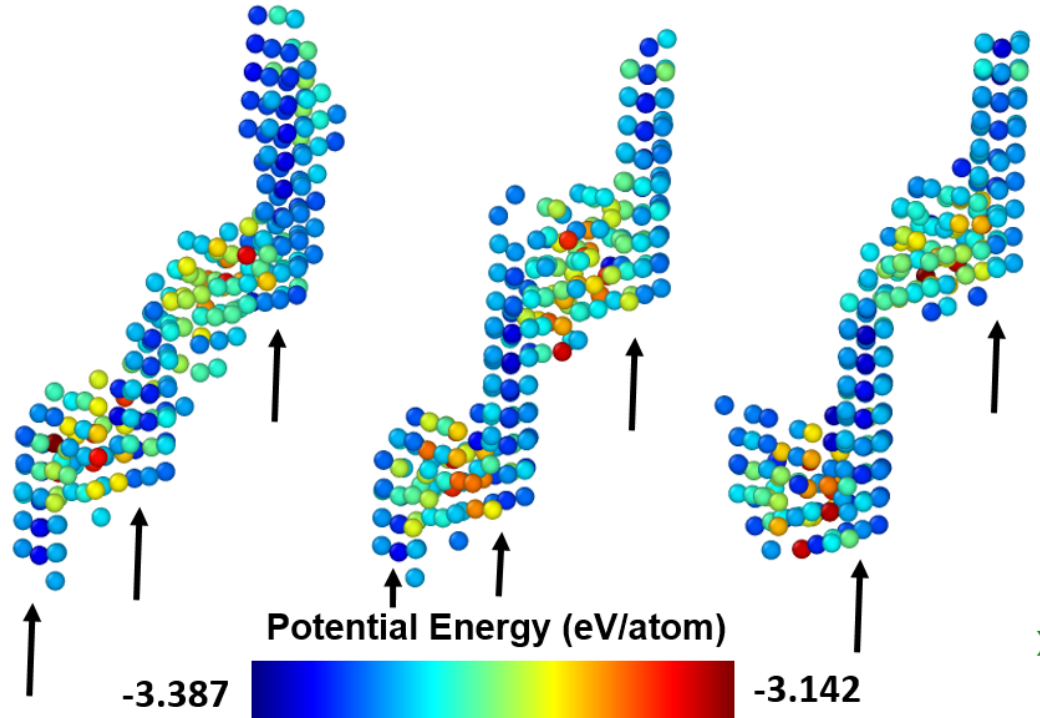

$-3.142$

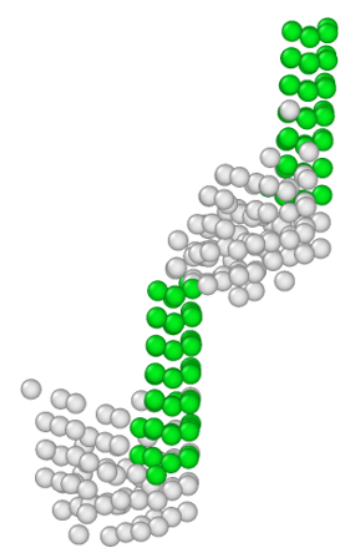

$\Sigma 1150.4^{\circ} / 3.7^{\circ}[110](\overline{1} 1 \overline{3} / \overline{1} 13)$

Figure 9. (a)-(c) The formation and evolution of selected special boundaries, with their $\Sigma$ type, disorientation, mis-misorientation, and boundary plane information listed below each image where applicable, during mechanical cycling of a $d=5 \mathrm{~nm}$ sample at $600 \mathrm{~K}$. (d)-(f) Cyclic loading drives a reduction in the energy the $\Sigma 11$ boundary through grain boundary faceting. (e) The individual facet planes along the $\Sigma 11$ boundary with low energy are identified as having a $\{113\}$ boundary plane normal. 
(a)

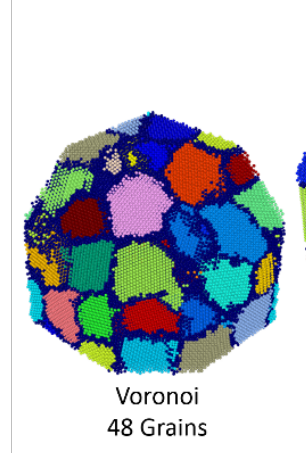

(b)

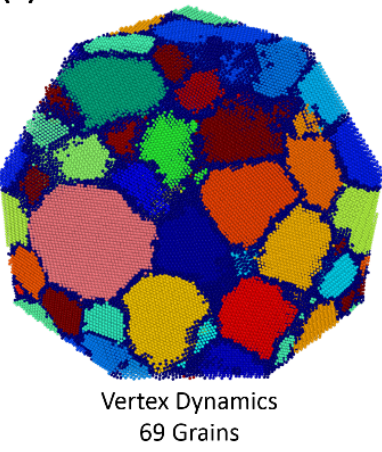

(c)

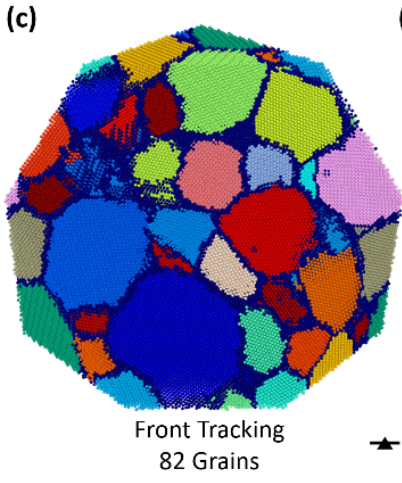

(d)

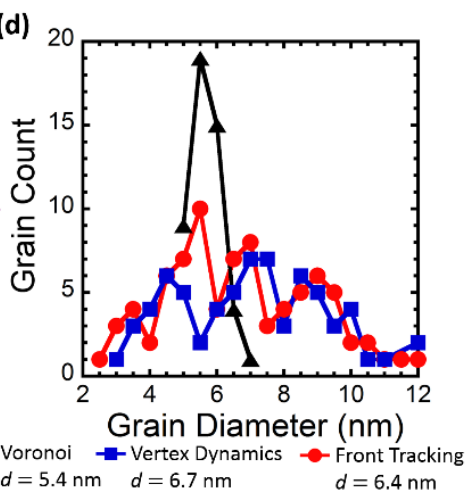

Figure 10. (a)-(c) Visual comparison of the three different starting configurations post annealing at $600 \mathrm{~K}$ with grains colored according to GTA identification. (d) The grain size distribution for all three samples before cycling was performed showing a much narrower distribution for the Voronoi sample. Calculated average grain size for each sample are also listed below the figure. 


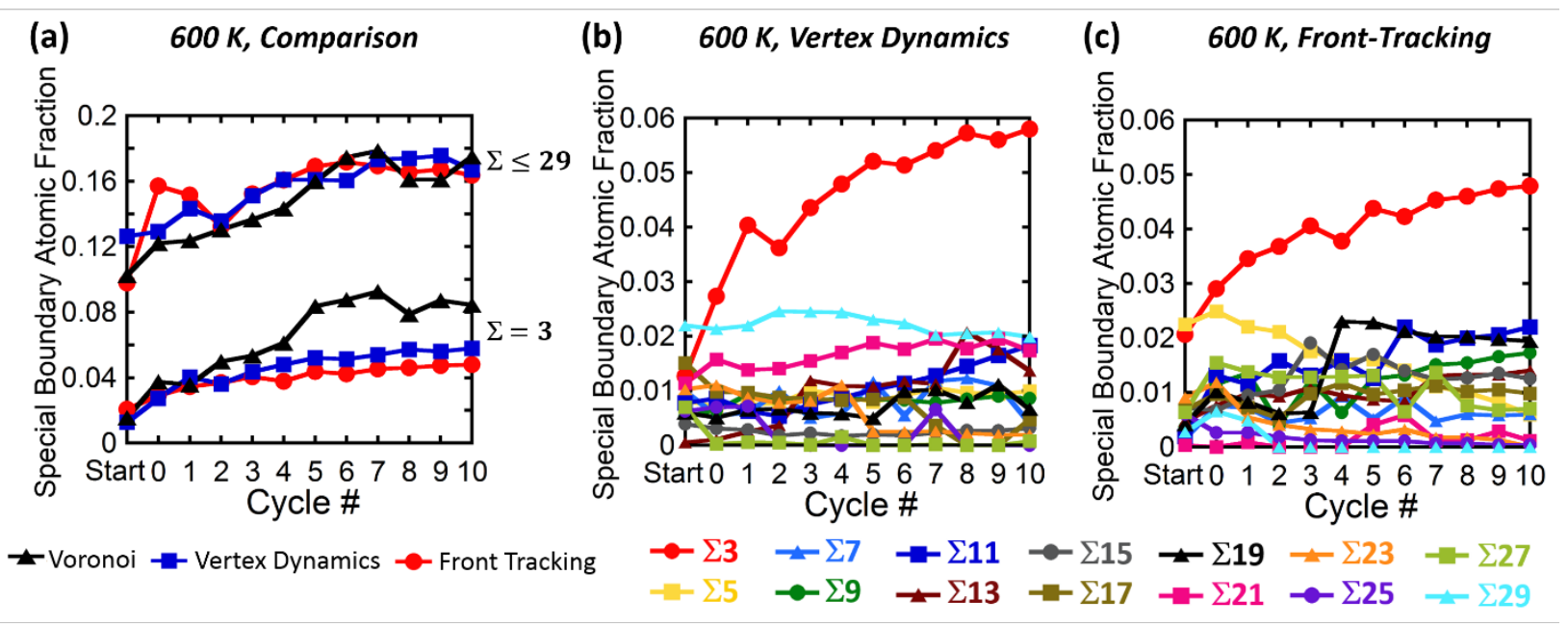

Figure 11. (a) The upper curves show the special boundary fraction evolution for all boundaries with $\Sigma \leq 29$ as a function of cycle number. The lower curves compare the difference in $\Sigma 3$ evolution for all three starting configurations. Detailed evolution of special boundary fraction for all special $\Sigma$ types is shown as a function of cycle number for the (b)Vertex Dynamics and (c) Front-Tracking samples, respectively. 


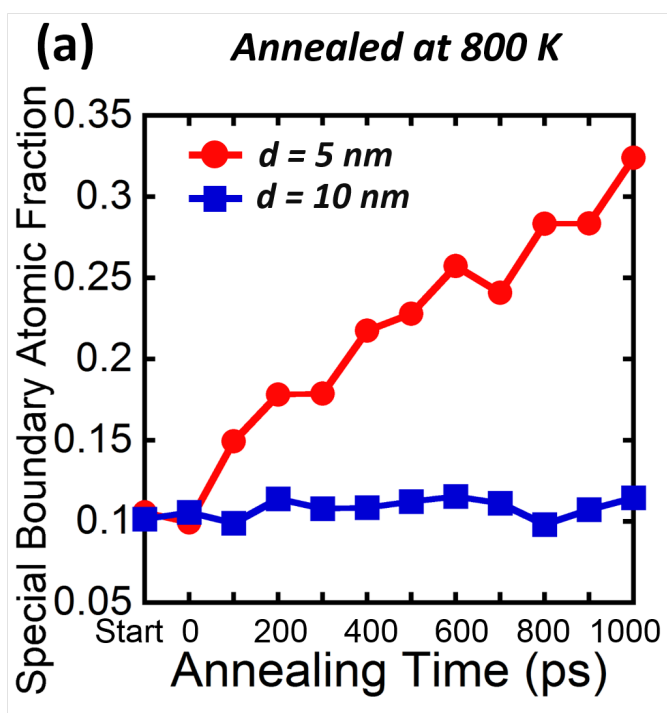

(b) $5 \mathrm{~nm}$, Annealed at $800 \mathrm{~K}$

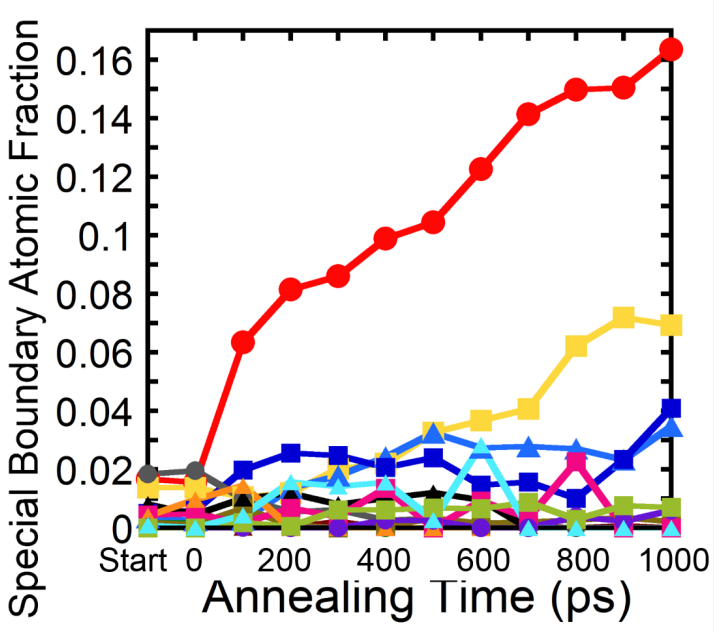

$$
\begin{aligned}
& -\Sigma 3 \multimap-\Sigma 9-\Sigma 15-\Sigma 21-\sum 27 \\
& -\sum 5 \rightarrow \Sigma 11 \rightarrow-\Sigma 17 \div \Sigma 23 \rightarrow \Sigma 29 \\
& \leftarrow \Sigma 7 \multimap \Sigma 13 \multimap \Sigma 19 \rightarrow \Sigma 25
\end{aligned}
$$

Figure 12. (a) Special boundary fraction for both grain sizes as a function of annealing time at 800 K. (b) A detailed breakdown of each special $\Sigma$ type for the $d=5 \mathrm{~nm}$ sample,showing that $\Sigma 3$ boundaries exhibit the fastest increase while $\Sigma 5, \Sigma 7$, and $\Sigma 11$ interfaces also become more common. 


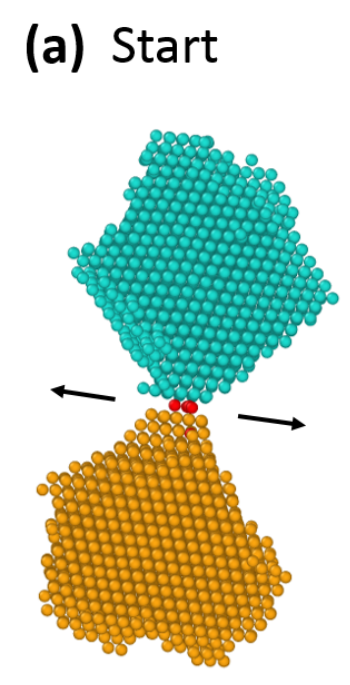

(b) 0 ps

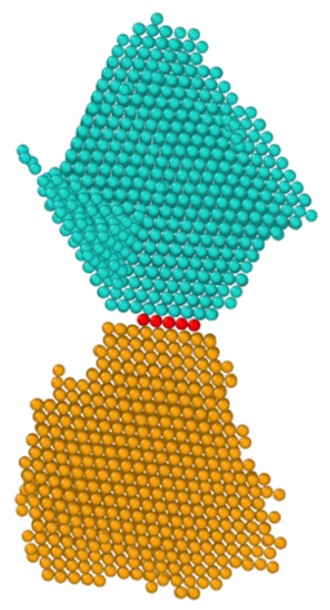

(c) $200 \mathrm{ps}$

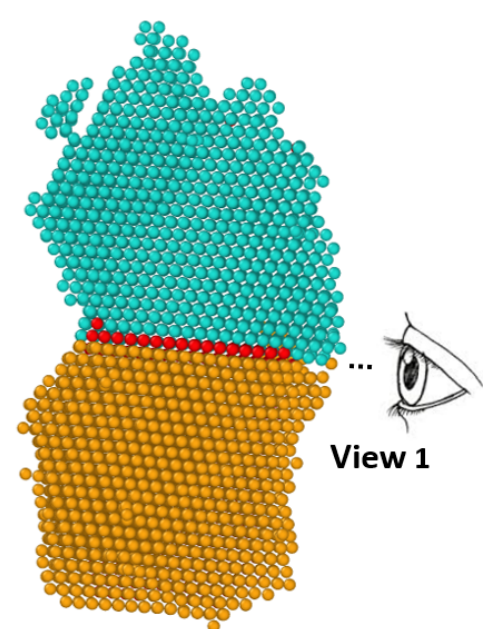

(e) 500 ps (View 1)

(d) 200 ps (View 1)

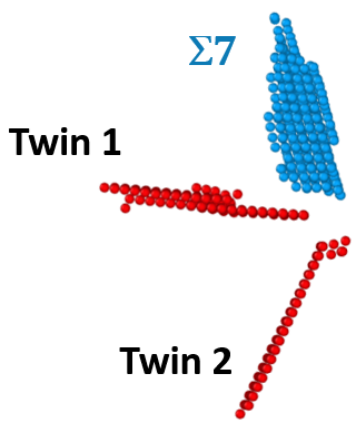

$\Sigma 737.58^{\circ} / 5.1^{\circ}[111]$

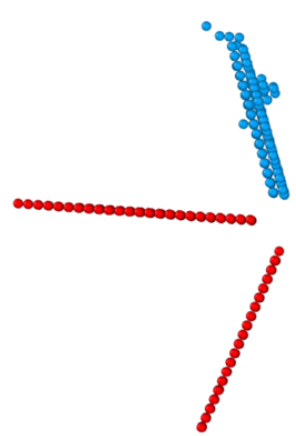

$\Sigma 735.42^{\circ} / 3.8^{\circ}[111](111) /(111)$

Figure 13.(a)-(c) Curvature-driven grain boundary migration leads to $\Sigma 3$ boundary lengthening in the $d=5 \mathrm{~nm}$ sample during annealing. Additional evidence of grain boundary facet removal is shown in (d) and (f), where a well-defined (111) plane forms along the $\Sigma 7$ boundary after 500 ps of annealing. 